TRANSACTIONS OF THE

AMERICAN MATHEMATICAL SOCIETY

Volume 355, Number 7, Pages 2675-2713

S 0002-9947(03)03121-0

Article electronically published on March 17, 2003

\title{
SYSTEMS OF DIAGONAL DIOPHANTINE INEQUALITIES
}

\author{
ERIC FREEMAN
}

\begin{abstract}
We treat systems of real diagonal forms $F_{1}(\mathbf{x}), F_{2}(\mathbf{x}), \ldots, F_{R}(\mathbf{x})$ of degree $k$, in $s$ variables. We give a lower bound $s_{0}(R, k)$, which depends only on $R$ and $k$, such that if $s \geq s_{0}(R, k)$ holds, then, under certain conditions on the forms, and for any positive real number $\epsilon$, there is a nonzero integral simultaneous solution $\mathbf{x} \in \mathbb{Z}^{s}$ of the system of Diophantine inequalities $\left|F_{i}(\mathbf{x})\right|<\epsilon$ for $1 \leq i \leq R$. In particular, our result is one of the first to treat systems of inequalities of even degree. The result is an extension of earlier work by the author on quadratic forms. Also, a restriction in that work is removed, which enables us to now treat combined systems of Diophantine equations and inequalities.
\end{abstract}

\section{INTRODUCTION}

1.1. Statement of main result. In 1980, Schmidt [17 proved a far-reaching result about systems of Diophantine inequalities of odd degree. Given any odd positive integers $d_{1}, \ldots, d_{R}$, Schmidt showed that there exists a positive integer $s_{1}=s_{1}\left(d_{1}, \ldots, d_{R}\right)$, depending only on $d_{1}, \ldots, d_{R}$, with the following property: given any positive integer $s \geq s_{1}$ and any real forms, or homogeneous polynomials, $G_{1}(\mathbf{x}), \ldots, G_{R}(\mathbf{x})$, in $s$ variables, of respective degrees $d_{1}, \ldots, d_{R}$, and given any positive number $\epsilon$, there always exists a nonzero integral vector $\mathbf{y} \in \mathbb{Z}^{s}$ satisfying the system

$$
\left|G_{1}(\mathbf{y})\right|<\epsilon,\left|G_{2}(\mathbf{y})\right|<\epsilon, \ldots,\left|G_{R}(\mathbf{y})\right|<\epsilon .
$$

So, in other words, as long as the forms are all of odd degree, and are defined in enough variables in terms only of the degrees, then there is a nonzero integral solution of the inequalities (1.1). Many particular classes of systems of the type (1.1) have been studied.

For Diophantine inequalities of even degree, the situation is much different. There is no such general result as above for integral solutions of Diophantine inequalities of even degree, and in fact there are few results at all for inequalities of even degree. (However, results are known if one allows solutions in algebraic integers in purely imaginary number fields. See Theorem 11.1 of [22].) In this article, we present one of the first results concerning systems of Diophantine inequalities of even degree, while at the same time removing a restriction from an earlier paper by the author, on quadratic Diophantine inequalities 10 . We are now able to remove

Received by the editors October 15, 2001.

2000 Mathematics Subject Classification. Primary 11D75; Secondary 11D41, 11D72, 11 P55.

Key words and phrases. Combined systems of Diophantine equations and inequalities, forms in many variables, applications of the Hardy-Littlewood method.

The author was supported by an NSF Postdoctoral Fellowship. 
the restriction by combining the powerful ideas of Bentkus and Götze [3] with the techniques of Nadesalingam and Pitman [16], and by adapting our previous work in [10] and [11] to treat the minor arcs properly.

To state our first result, we require some notation and definitions. We shall be working with systems of diagonal forms $F_{i}(\mathbf{x})$ given by

$$
F_{i}(\mathbf{x})=\lambda_{i 1} x_{1}^{k}+\lambda_{i 2} x_{2}^{k}+\ldots+\lambda_{i s} x_{s}^{k} \quad(1 \leq i \leq R) .
$$

For systems of forms $F_{i}$ as in (1.2), we define the coefficient matrix of the system $\mathbf{F}$ to be the matrix

$$
A=\left(\lambda_{i j}\right)_{\substack{1 \leq i \leq R \\ 1 \leq j \leq s}}
$$

For $1 \leq j \leq s$, we denote the $j^{\text {th }}$ column of $A$ by $\boldsymbol{\lambda}_{j}$.

Now suppose that $J$ is a subset of the set of indices $\{1,2, \ldots, s\}$. We define $A_{J}$ to be the submatrix of $A$ consisting of the columns $\boldsymbol{\lambda}_{j}$ with $j \in J$, and we define $r\left(A_{J}\right)$ to be the rank of the matrix $A_{J}$. Finally, if $\mathbf{x} \in \mathbb{R}^{s}$ satisfies $F_{i}(\mathbf{x})=0$ for $1 \leq i \leq R$ and the matrix

$$
\left(\frac{\partial F_{i}}{\partial x_{j}}\right)_{\substack{1 \leq i \leq R \\ 1 \leq j \leq s}}
$$

is of full rank, then we say that $\mathbf{x}$ is a nonsingular solution of the system $\mathbf{F}$.

Now, for integers $R$ and $k$ and any real number $u$, we define the functions

$$
m_{0}(R, k, u)=\left\{\begin{array}{cl}
\min \left(4 R^{2}+4 R+1,384 \log 16 R+5\right) & \text { if } k=2 \\
\frac{R k \log 2 k}{\log 2}+u k \log (R \log 2 k) & \text { if } k \text { is odd and } k \geq 3 \\
k\left[48 k^{2} \log 3 R k^{2}\right] & \text { if } k \geq 3,
\end{array}\right.
$$

and

$$
n_{0}(k, u)=\left\{\begin{array}{cl}
5 & \text { if } \quad k=2 \\
\min \left(2^{k}+1, k(\log k+\log \log k+2)+\frac{u k \log \log k}{\log k}\right) & \text { if } \quad k \geq 3 .
\end{array}\right.
$$

We can now state our first result.

Theorem 1.1. Suppose that $k$ is an integer with $k \geq 2$ and that $R$ is a positive integer. There are absolute real positive constants $\tilde{C}_{1}$ and $\tilde{C}_{2}$ for which the following property holds:

Suppose that $\ell$ is an integer satisfying

$$
\ell \geq \max \left(m_{0}\left(R, k, \tilde{C}_{1}\right), n_{0}\left(k, \tilde{C}_{2}\right)\right) .
$$

Suppose that $s$ is an integer satisfying $s \geq \ell R$. For $1 \leq i \leq R$, suppose that $F_{i}(\mathbf{x})$ is a real diagonal form of degree $k$, as in (1.2). Let $A$ be the coefficient matrix of the system $\mathbf{F}$, as in (1.3). Assume that the following two conditions are satisfied:

(i) Either $k$ is odd, or there exists a real nonsingular solution $\mathbf{y}$ of the system

$$
F_{1}(\mathbf{y})=F_{2}(\mathbf{y})=\cdots=F_{R}(\mathbf{y})=0 .
$$

(ii) For every subset $J \subseteq\{1,2, \ldots, s\}$, one has $|J| \leq s-\ell\left(R-r\left(A_{J}\right)\right)$.

Fix any positive real number $\epsilon$. Then there is a nonzero integral solution $\mathbf{x} \in \mathbb{Z}^{s}$ of the system

$$
\left|F_{i}(\mathbf{x})\right|<\epsilon \quad \text { for } \quad 1 \leq i \leq R
$$


For general even $k$, Theorem 1.1 is one of the first results of its kind to our knowledge. We note that at least some conditions similar to (i) and (ii) are necessary, as may be seen by considering the examples given after Theorem 2 of [7].

For odd $k$, we note that Theorem 1.1 is not very much of an improvement beyond that given by Nadesalingam and Pitman [16, and could presumably be obtained by combining their methods with results of Vaughan 19], [20] and work of Wooley [23. We also observe that, following the method of Section 7.2 of [16], we could remove condition (ii) for odd $k$ if we chose to do so.

As well, our method of proof shows that under the conditions of Theorem 1.1, we can give a lower bound of the expected order of magnitude, $P^{s-R k}$, for the number of solutions of (1.7) in a box of size $P$, for all sufficiently large $P$. This was not previously known, even in the special case of systems of inequalities of odd degree. We emphasize that when using our methods, condition (ii) is necessary to obtain this lower bound. Presumably, one could also give an asymptotic formula for the number of solutions, by combining with the methods of [12].

Note that we have excluded the case $k=1$ from the statement of the theorem. In this case, our knowledge is much better. For $k=1$, if one has $s \geq R+1$, a nonzero solution of (1.7) may be found using a box principle, whether or not condition (ii) holds. (See the Lemma in 4].) For $k=1$, one can also find many solutions of (1.7) in a box of size $P$. (See Lemma 1 of 9 .)

1.2. Combined systems of Diophantine equations and inequalities. We note now that it would actually be fairly routine to give at least one result on inequalities of even degree; one could simply generalize the work in [10] by combining those techniques with the methods in 11]. However, such a generalization would exclude many important classes of systems of inequalities, for example those in which some of the forms are integral. We were forced to exclude such systems in [10] because of the methods we used. In our current work, we are able to treat these formerly excluded systems. We give some background to more fully explain.

In [10, we considered simultaneous systems of diagonal quadratic Diophantine inequalities. For a positive integer $R$, define, for $1 \leq i \leq R$, the real quadratic forms

$$
Q_{i}(\mathbf{x})=\lambda_{i 1} x_{1}^{2}+\lambda_{i 2} x_{2}^{2}+\ldots+\lambda_{i s} x_{s}^{2} .
$$

It was proved in 10 that for every positive real number $\epsilon$, under certain conditions on the system of forms $Q_{1}, Q_{2}, \ldots, Q_{R}$, there is an integral vector $\mathbf{x} \in \mathbb{Z}^{s} \backslash\{\mathbf{0}\}$ such that one has

$$
\left|Q_{i}(\mathbf{x})\right|<\epsilon \quad(1 \leq i \leq R) .
$$

In that paper, one of the conditions we assumed was the following. (See condition (iii) of Theorem 3 of [10].)

For each choice of $\left(\beta_{1}, \beta_{2}, \ldots, \beta_{R}\right) \in \mathbb{R}^{R} \backslash\{\mathbf{0}\}$, there is at least one coefficient of $\beta_{1} Q_{1}+\beta_{2} Q_{2}+\ldots+\beta_{R} Q_{R}$ that is irrational.

This condition allowed us to use a modification of the remarkable work of Bentkus and Götze [3, but excludes certain important systems from consideration. The restriction (1.8) rules out systems in which one or more of the forms is an integral form, and also any system in which any nontrivial linear combination of the forms 
is an integral form. The central new contribution of this paper is in removing the condition (1.8).

We now state a more technical and more general version of Theorem [1.1. We require more notation. For a real vector $\boldsymbol{\beta}=\left(\beta_{1}, \ldots, \beta_{R}\right) \in \mathbb{R}^{R}$ and a system $\mathbf{G}$ of forms $G_{1}(\mathbf{x}), G_{2}(\mathbf{x}), \ldots, G_{R}(\mathbf{x})$, we define the form

$$
(\boldsymbol{\beta} \cdot \mathbf{G})(\mathbf{x})=\beta_{1} G_{1}(\mathbf{x})+\beta_{2} G_{2}(\mathbf{x})+\ldots+\beta_{R} G_{R}(\mathbf{x}) .
$$

Also, for real numbers $x$, we define

$$
e(x)=e^{2 \pi i x} .
$$

Theorem 1.2. Suppose that $k$ is an integer with $k \geq 2$ and that $r$ and $R$ are integers with $R \geq 1$ and $0 \leq r \leq R$. Then there are absolute positive real constants $\tilde{C}_{1}$ and $\tilde{C}_{2}$ with the following property:

Define $n_{0}(k, u)$ as in (1.5). Suppose that $\ell$ is an integer satisfying

$$
\ell \geq n_{0}\left(k, \tilde{C}_{2}\right) \text {. }
$$

Let $s$ be an integer with $s \geq \ell R$. Also suppose, for $1 \leq i \leq R$, that

$$
F_{i}(\mathbf{x})=\lambda_{i 1} x_{1}^{k}+\lambda_{i 2} x_{2}^{k}+\ldots+\lambda_{i s} x_{s}^{k}
$$

is a diagonal form with real coefficients. Let $A$ be the coefficient matrix of the system $\mathbf{F}$, as in $(\underline{1.3})$.

Assume that the following four conditions are satisfied:

(i) Either $k$ is odd, or there exists a real nonsingular solution $\mathbf{y}$ of the system

$$
F_{1}(\mathbf{y})=F_{2}(\mathbf{y})=\cdots=F_{R}(\mathbf{y})=0 .
$$

(ii) For every subset $J \subseteq\{1,2, \ldots, s\}$, one has $|J| \leq s-\ell\left(R-r\left(A_{J}\right)\right)$.

(iii) The forms $F_{1}, F_{2}, \ldots, F_{r}$ have integer coefficients; also, if $\boldsymbol{\alpha}=\left(\alpha_{1}, \alpha_{2}, \ldots\right.$, $\left.\alpha_{R}\right) \in \mathbb{R}^{R}$ and $\boldsymbol{\alpha} \cdot \mathbf{F}$ is a rational form, then $\alpha_{r+1}=\alpha_{r+2}=\cdots=\alpha_{R}=0$.

(iv) If $r \geq 1$ holds, then there is a positive real constant $c(\mathbf{F})$ such that one has

$$
\mathfrak{S}=\sum_{q=1}^{\infty} \sum_{\substack{\mathbf{a}:\left(a_{1}, \ldots, a_{r}, q\right)=1 \\ 1 \leq a_{i} \leq q(1 \leq i \leq r)}} q^{-s} \prod_{j=1}^{s} \sum_{x=1}^{q} e\left(\frac{x^{k}}{q} \sum_{i=1}^{r} \lambda_{i j} a_{i}\right) \geq c(\mathbf{F}) .
$$

Fix any positive real number $\epsilon$. Then there is a nonzero integral solution $\mathbf{x} \in \mathbb{Z}^{s}$ of the system

$$
\begin{aligned}
F_{i}(\mathbf{y})=0 & \text { for } & 1 \leq i \leq r \\
\left|F_{i}(\mathbf{y})\right|<\epsilon & \text { for } & r+1 \leq i \leq R .
\end{aligned}
$$

Moreover, if we define $m_{0}(r, k, u)$ as in (1.4) and we assume that the condition $\ell \geq m_{0}\left(r, k, \tilde{C}_{1}\right)$ holds, then we may omit condition (iv) from our assumptions.

Some discussion of the condition (iii) is warranted here, since it is the most important distinction between Theorems 1.1 and 1.2. In Theorem 1.1, we consider systems of inequalities (1.7). Now, if one chooses $\epsilon<1$, and $F_{1}(\mathbf{x})$, say, is actually an integral form, then the system (1.7) reduces to the system

$$
\begin{aligned}
F_{1}(\mathbf{y}) & =0, \\
\left|F_{i}(\mathbf{y})\right| & <\epsilon \quad \text { for } \quad 2 \leq i \leq R .
\end{aligned}
$$


So, in this case, the system of $R$ inequalities actually reduces to a system of one equation and $R-1$ inequalities. A similar reduction occurs if some nontrivial real linear combination of the forms $F_{i}$, say $\alpha_{1} F_{1}+\alpha_{2} F_{2}+\ldots+\alpha_{R} F_{R}$, is an integral form. In these situations, one might say that there is actually an equation hidden in the system of inequalities. The condition (iii) ensures that there are actually $r$ equations in the system and $R-r$ "true" inequalities. It is helpful to ensure that there are not any more "hidden" equations because it turns out that one requires more variables to treat the system if there are more equations present. One may think of Theorem 1.2, more or less, as the sub-case of Theorem 1.1 in which there are exactly $r$ equations present in a system of $R$ inequalities. We note that the second clause of condition (iii) is vacuous if $r=R$ holds.

One might question if, in condition (iii), the term $\boldsymbol{\alpha} \cdot \mathbf{F}$ could be replaced by the term $\alpha_{r+1} F_{r+1}+\alpha_{r+2} F_{r+2}+\ldots+\alpha_{R} F_{R}$, to give a slightly weaker condition asserting that $r$ equations are "hidden" in the system. It turns out that one can not, as may be seen by considering the example

$$
\begin{aligned}
F_{1}(\mathbf{x})=2 x_{1}^{k}+3 x_{2}^{k}+a_{3} x_{3}^{k}+a_{4} x_{4}^{k}+\ldots+a_{s} x_{s}^{k} \\
F_{2}(\mathbf{x})=(2+\sqrt{2}) x_{1}^{k}+(3+\sqrt{2}) x_{2}^{k}+\left(a_{3}+\sqrt{2}\right) x_{3}^{k} \\
+\left(a_{4}+\sqrt{2}\right) x_{4}^{k}+\ldots+\left(a_{s}+\sqrt{2}\right) x_{s}^{k},
\end{aligned}
$$

where $a_{3}, a_{4}, \ldots, a_{s}$ are any integers. Here, for $r=1$ and $R=2$, condition (iii) does not hold, since $(1 / \sqrt{2})\left(F_{2}-F_{1}\right)$ is an integral form, and thus the system (1.10) is equivalent in this case, for small $\epsilon$, to the system

$$
F_{1}(\mathbf{x})=\frac{1}{\sqrt{2}}\left(F_{2}(\mathbf{x})-F_{1}(\mathbf{x})\right)=0
$$

on the other hand, for any nonzero real number $\alpha_{2}$, the form $\alpha_{2} F_{2}$ is not a rational form since the ratio $((3+\sqrt{2}) /(2+\sqrt{2}))$ is irrational; so this system does not satisfy the suggested replacement condition. So the putative replacement condition is not strong enough.

We now discuss condition (iv). The term $\mathfrak{S}$ is the so-called singular series, and condition (iv) simply states that it is bounded below by a positive constant, a necessary precondition when using the Hardy-Littlewood method. As the last sentence of Theorem 1.2 states, we could have omitted the condition from our assumptions in favor of a lower bound for $\ell$. However, since the consideration of the singular series is not our central focus in this work, we have chosen to include condition (iv) so that our result can be improved immediately and transparently if improvements arise concerning the singular series and the $p$-adic problem. This should certainly be possible in the case $k=2$, for example. Also, we wish to clearly indicate that the condition $\ell \geq m_{0}\left(r, k, \tilde{C}_{1}\right)$ is needed only because of the $p$-adic problem.

1.3. Related results. We now compare our work with other results. For even $k$, Theorem 1.1 is an analogue of a result of Davenport and Lewis, concerning systems of Diophantine equations of even degree. (See Theorem 2 of [7].) They assume that 
a system of diagonal equations

$$
F_{1}(\mathbf{x})=F_{2}(\mathbf{x})=\cdots=F_{R}(\mathbf{x})=0,
$$

of even degree $k$, with $k>2$, has a real nonsingular solution and also that for $1 \leq S \leq R$, every set of $S$ independent integral linear combinations of $F_{1}, \ldots, F_{R}$ contains at least

$$
\left[48 R S k^{2} \log \left(3 R k^{2}\right)\right]
$$

variables that appear explicitly. Under these conditions, the system of equations has a nonzero integral solution. We note that if one replaces the quantity in (1.11) by $S \ell$, and restricts to integral forms, then one can show that their second condition is equivalent to condition (ii) of Theorem 1.1

Nadesalingam and Pitman 16 proved that any $R$ real diagonal Diophantine inequalities of odd degree $k$, with $k \geq 13$, in $s$ variables with

$$
s \geq 3 R^{2} k^{2} \log (3 R k)
$$

have a nonzero solution. We note that they do not require any condition that is similar to condition (ii) of Theorem 1.1. Also, we observe that they could certainly have used their methods to obtain similar results, although with a different lower bound for $s$, in the cases $k<13$, but in order to streamline the presentation they did not do so.

Finally, we note that Brüdern and Cook 5 have given a result on systems of diagonal Diophantine inequalities of odd degree. Under certain conditions on the coefficient matrix of the system, they show that there is a nonzero solution of the system of inequalities. They require an assumption similar to condition (ii) of Theorem 1.1 and also a condition that is stronger than (1.8). The number of variables they require is on the order of $R n_{0}\left(k, \tilde{C}_{2}\right)$. We also note that they can find a lower bound of the expected order of magnitude for the number of solutions of their system in a box of size $P$ for a sequence of positive $P$ tending to infinity, although not for all large $P$, as our treatment provides.

1.4. Methods used. The general strategy of the proof is to combine the method of Bentkus and Götze [3], which is very effective for Diophantine inequalities, with the techniques that Nadesalingam and Pitman [16] use to treat combined systems of Diophantine equations and inequalities. We remark that the techniques of Nadesalingam and Pitman are themselves a combination of the Hardy-Littlewood method and the Davenport-Heilbronn method. Using the techniques of Nadesalingam and Pitman allows us to treat those systems of inequalities that contain "hidden" equations. For those who are familiar with their argument, we note that we do not have a so-called residual set in our proof, as in their paper.

One other crucial result is needed, and this involves showing that, on the minor arcs, our exponential sums are smaller than the trivial bound. In previous work on these types of problems, including [10], [11, and essentially also [3], such a result was achieved by splitting the minor arcs into two regions and handling each separately. In this paper, we handle both of these regions together, which is not only cleaner, but also seems to be necessary here.

I would like to thank Scott Parsell for showing me how to improve Lemma 6.1 I would also like to thank Michael Knapp and Professor Wooley for indicating to me how to prove part of Lemma 8.5 . 


\section{Deduction of Theorem 1.1 from Theorem 1.2}

The bulk of this paper is dedicated to proving Theorem 1.2. In this section, however, we demonstrate how Theorem 1.2 implies Theorem 1.1 To this end, we consider a system $\mathbf{F}$ of real diagonal forms $F_{1}, F_{2}, \ldots, F_{R}$ as in Theorem 1.1

We give a definition first. Suppose that $G_{1}, G_{2}, \ldots, G_{R}$ and $H_{1}, H_{2}, \ldots, H_{R}$ are two systems of forms. If there exists a set of $R$ linearly independent real vectors $\boldsymbol{\beta}_{1}, \boldsymbol{\beta}_{2}, \ldots, \boldsymbol{\beta}_{R} \in \mathbb{R}^{R}$ such that

$$
H_{i}(\mathbf{x})=\boldsymbol{\beta}_{i} \cdot \mathbf{G} \quad \text { for } \quad 1 \leq i \leq R,
$$

then we say that the system $\mathbf{H}$ is equivalent to the system $\mathbf{G}$, which we denote by $\mathbf{G} \sim \mathbf{H}$. It is easy to check that this is in fact an equivalence relation. We observe as well that if $\mathbf{G}$ is, in particular, a system of diagonal forms, and $\mathbf{G} \sim \mathbf{H}$ holds, then $\mathbf{H}$ is also a system of diagonal forms.

For any system of forms $\mathbf{G}$, we define $z(\mathbf{G})$ to be the number of forms among $G_{1}, G_{2}, \ldots, G_{R}$ that are integral, that is, whose coefficients are all integers. Now for our system of forms $\mathbf{F}$, we define

$$
r=r(\mathbf{F})=\max _{\mathbf{G} \sim \mathbf{F}} z(\mathbf{G}) .
$$

In other words, $r(\mathbf{F})$ is simply the maximum number of forms that are integral in any system $\mathbf{G}$ equivalent to $\mathbf{F}$. We clearly have $0 \leq r \leq R$.

Now suppose that $\mathbf{G}$ is a system equivalent to $\mathbf{F}$ and that $\mathbf{G}$ has $r$ integral forms. So there exist $R$ real linearly independent vectors $\boldsymbol{\beta}_{1}, \boldsymbol{\beta}_{2}, \ldots, \boldsymbol{\beta}_{R} \in \mathbb{R}^{R}$ such that $G_{i}=\boldsymbol{\beta}_{i} \cdot \mathbf{F}$ for $1 \leq i \leq R$, and also the system $\mathbf{G}$ contains $r$ integral forms. By relabeling if necessary, we may assume that $G_{1}, G_{2}, \ldots, G_{r}$ are integral forms. We now show that conditions (i)-(iv) of Theorem 1.2 hold for this system $\mathbf{G}$. Then we will apply Theorem 1.2 to $\mathbf{G}$, and we will see that the nonzero solution of the system $\mathbf{G}$ is also, under certain conditions, a solution of the system $\mathbf{F}$.

Since $\mathbf{F}$ is equivalent to $\mathbf{G}$, if the coefficient matrix of $\mathbf{F}$ is $A$, then the coefficient matrix of $\mathbf{G}$ is $T A$ for the nonsingular $R \times R$ matrix $T$ with rows $\boldsymbol{\beta}_{i}$. Thus, for any subset $J \subseteq\{1,2, \ldots, s\}$, we have

$$
r\left((T A)_{J}\right)=r\left(T A_{J}\right)=r\left(A_{J}\right) .
$$

The system $\mathbf{F}$ has a nonsingular solution $\mathbf{x}$ if and only if there is a subset $J$ of $\{1,2, \ldots, s\}$ with $|J|=R$ satisfying $r\left(A_{J}\right)=R$ and $\prod_{j \in J} x_{j} \neq 0$. Thus the existence of a real nonsingular solution for $\mathbf{G}$ follows from the existence of such a solution for F. So condition (i) holds for G. By (2.1), it is easy to see that condition (ii) holds for the coefficient matrix of $\mathbf{G}$, because it holds for the coefficient matrix of $\mathbf{F}$.

Now we turn to showing that condition (iii) of Theorem 1.2 holds for the system G. To this end, suppose that $\boldsymbol{\alpha}^{\prime}=\left(\alpha_{1}^{\prime}, \alpha_{2}^{\prime}, \ldots, \alpha_{R}^{\prime}\right) \in \mathbb{R}^{R}$ is a real vector such that $\boldsymbol{\alpha}^{\prime} \cdot \mathbf{G}$ is a rational form. We need to show that $\alpha_{r+1}^{\prime}=\alpha_{r+2}^{\prime}=\cdots=\alpha_{R}^{\prime}=0$ holds. This holds vacuously if $r=R$. For $r<R$, clearing denominators, we see that there is a nonzero integer $n$ such that, defining $\boldsymbol{\alpha}=n \boldsymbol{\alpha}^{\prime}$, we have

$$
\boldsymbol{\alpha} \cdot \mathbf{G}=n \boldsymbol{\alpha}^{\prime} \cdot \mathbf{G} \in \mathbb{Z}[\mathbf{x}] .
$$

Since $n$ is nonzero, to prove that $\alpha_{r+1}^{\prime}=\alpha_{r+2}^{\prime}=\cdots=\alpha_{R}^{\prime}=0$ holds, it is enough to prove that we have $\alpha_{r+1}=\alpha_{r+2}=\cdots=\alpha_{R}=0$.

So suppose that this is not the case. Letting $\mathbf{e}_{1}, \mathbf{e}_{2}, \ldots, \mathbf{e}_{R}$ be the standard unit basis for $\mathbb{R}^{R}$, we then have that $\mathbf{e}_{1}, \mathbf{e}_{2}, \ldots, \mathbf{e}_{r}, \boldsymbol{\alpha}$ are $r+1$ linearly independent 
vectors in $\mathbb{R}^{R}$. We may thus extend this set to a basis, say $\gamma_{1}, \gamma_{2}, \ldots, \gamma_{R}$ of $\mathbb{R}^{R}$, with $\gamma_{i}=\mathbf{e}_{i}$ for $1 \leq i \leq r$, and $\gamma_{r+1}=\boldsymbol{\alpha}$. Then the system of forms $\gamma_{1} \cdot \mathbf{G}, \gamma_{2} \cdot \mathbf{G}, \ldots, \gamma_{R} \cdot \mathbf{G}$ is equivalent to $\mathbf{G}$, and thus in turn to $\mathbf{F}$. But its first $r+1$ forms are integral. This contradicts the definition of $r(\mathbf{F})$, and thus we must in fact have

$$
\alpha_{r+1}=\alpha_{r+2}=\cdots=\alpha_{R}=0 .
$$

Thus condition (iii) holds for $\mathbf{G}$.

Now, since $\ell$ satisfies (1.6), we have $\ell \geq m_{0}\left(R, k, \tilde{C}_{1}\right)$, and thus we certainly have $\ell \geq m_{0}\left(r, k, \tilde{C}_{1}\right)$, whence by the final sentence of Theorem 1.2, condition (iv) is unnecessary. Since we have also seen that conditions (i)-(iii) of Theorem 1.2 hold, we may apply Theorem 1.2 to the system $\mathbf{G}$.

Before doing so, we give some more notation. For a vector $\mathbf{x}=\left(x_{1}, x_{2}, \ldots, x_{s}\right) \in$ $\mathbb{R}^{s}$, define

$$
|\mathbf{x}|=\max _{1 \leq j \leq s}\left|x_{j}\right| .
$$

For an $R \times s$ matrix $M=\left(m_{i j}\right)_{\substack{1 \leq i \leq R \\ 1 \leq j \leq s}}$, we define

$$
\|M\|=\max _{\substack{1 \leq i \leq R \\ 1 \leq j \leq s}}\left|m_{i j}\right| .
$$

We note that the notation differs slightly from that used by some other authors, for example Nadesalingam and Pitman [16]. Similarly, for a system F as in (1.2), we define

$$
\|\mathbf{F}\|=\max _{\substack{1 \leq i \leq R \\ 1 \leq j \leq s}}\left|\lambda_{i j}\right|
$$

Defining the matrix $T$ as above, we certainly have $\operatorname{det}(T) \neq 0$ and $\|T\| \neq 0$; so we may apply Theorem 1.2 to the system $\mathbf{G}$ with $\epsilon$ replaced by the quantity $(|\operatorname{det}(T)| \epsilon) /\left(R^{R}\|T\|^{R-1}\right)$. We obtain a nonzero integral solution $\mathbf{x} \in \mathbb{Z}^{s}$ of the system

$$
\left|G_{i}(\mathbf{x})\right|<\frac{|\operatorname{det}(T)| \epsilon}{R^{R}\|T\|^{R-1}} \quad(1 \leq i \leq R) .
$$

By Cramer's rule and Hadamard's rule, it follows that $\mathbf{x}$ is also a solution of the system (1.7), whence Theorem 1.1 follows.

We now turn to the proof of Theorem[1.2, which comprises the rest of the paper.

\section{Initial Reductions}

In this section, we reduce the problem of proving Theorem 1.2 to the consideration of a system of forms as in Theorem 1.2, but under a few more restrictions. This will make our application of the Hardy-Littlewood method easier. We first note that by considering the forms $\epsilon^{-1} F_{i}$, it is enough to consider only the case

$$
\epsilon=1 \text {. }
$$

We can also assume that we have

$$
\|\mathbf{F}\| \geq 1
$$

If this were not the case, then $(1,0,0, \ldots, 0)$ would be a solution of the system (1.10) and we would be done. 
We now quote a lemma, which seems to have been first used in this field by Low, Pitman and Wolff. (See Lemma 1 of [13].) It is actually a special case of a result on matroids, apparently due originally to Edmonds [8]. A proof can also be found in Aigner. (See Proposition 6.45 of [1.)

Lemma 3.1. Let $A$ be an $R \times s$ matrix over a field $K$ and let $w$ be a positive integer. The matrix $A$ has an $R \times R w$ partitionable submatrix (that is, $A$ includes $w$ disjoint $R \times R$ submatrices that are nonsingular over $K$ ) if and only if the following condition is satisfied:

$$
|J| \leq s-w\left(R-r\left(A_{J}\right)\right) \quad \text { for all subsets } J \subseteq\{1,2, \ldots, s\} .
$$

To be clear, by including $w$ disjoint $R \times R$ nonsingular submatrices, we mean that there is some permutation of the columns so that the first $R$ columns form a nonsingular matrix, as do the second $R$ columns, and so on.

Note that the condition (3.1) is exactly condition (ii) of Theorem 1.2 in the case $w=\ell$. Thus we may apply the lemma to the coefficient matrix $A$ of the system $\mathbf{F}$, with the choice $w=\ell$. Therefore, $A$ has an $R \times R \ell$ partitionable submatrix. By relabeling variables if necessary, we may write

$$
A=\left[\begin{array}{llllllll}
A_{1} & A_{2} & \ldots & A_{\ell} & \boldsymbol{\lambda}_{\ell R+1} & \lambda_{\ell R+2} & \ldots & \boldsymbol{\lambda}_{s}
\end{array}\right],
$$

where $A_{v}$ is an $R \times R$ submatrix for $1 \leq v \leq \ell$ and where

$$
\Delta_{v}=\left|\operatorname{det}\left(A_{v}\right)\right| \neq 0 \quad \text { for } \quad 1 \leq v \leq \ell .
$$

Now consider the system $\mathbf{F}$ in the case that $k$ is odd. We show that $\mathbf{F}$ has a real nonsingular solution. Since $A$ has the form (3.2), and (3.3) holds, one can see that $\mathbf{F}$ is equivalent to a system $\mathbf{G}$ with coefficient matrix $B$ such that the left-hand $R \times 2 R$ submatrix of $B$ has the form

$$
\left[\begin{array}{ll}
I & B_{2}
\end{array}\right]
$$

here $I$ is the $R \times R$ identity matrix, and $B_{2}$ is a nonsingular $R \times R$ matrix. We can find real numbers $z_{R+1}, z_{R+2}, \ldots, z_{2 R}$ satisfying

$$
B_{2}\left[\begin{array}{c}
z_{R+1} \\
z_{R+2} \\
\vdots \\
z_{2 R}
\end{array}\right]=\left[\begin{array}{c}
-1 \\
-1 \\
\vdots \\
-1
\end{array}\right] .
$$

Now let $z_{j}=1$ for $1 \leq j \leq R$, and let $z_{j}=0$ for $j>2 R$. Then for $1 \leq j \leq s$, define $x_{j}=z_{j}^{1 / k}$, which is always real, since $k$ is odd. Setting $\mathbf{x}=\left(x_{1}, x_{2}, \ldots, x_{s}\right)$, one can observe that $G_{i}(\mathbf{x})=0$ for $1 \leq i \leq R$. Now the left-hand $R \times R$ matrix of $\left(\frac{\partial G_{i}}{\partial x_{j}}\right)_{\substack{1 \leq i \leq R \\ 1 \leq j \leq s}}$ has determinant $k^{R} x_{1}^{k-1} x_{2}^{k-1} \cdots x_{R}^{k-1}$, which is nonzero. Thus the system $\mathbf{G}$ has a real nonsingular solution, whence, as in Section 2 the system $\mathbf{F}$ does as well.

Thus, whether $k$ is odd or even, we know that there is a nonsingular solution of the system

$$
F_{i}(\mathbf{x})=0 \quad(1 \leq i \leq R) .
$$

We now show that there is a real nonsingular solution whose components are all positive. As noted above, there is a subset $J=\left\{j_{1}, j_{2}, \ldots, j_{R}\right\} \subseteq\{1,2, \ldots, s\}$ with 
$|J|=R$ and a real vector $\mathbf{x} \in \mathbb{R}^{s}$ such that we have $\operatorname{det}\left(A_{J}\right) \neq 0$ and $\prod_{j \in J} x_{j} \neq 0$. Now, for $1 \leq i \leq R$, we define the linear form

$$
L_{i}(\mathbf{y})=L_{i}\left(y_{1}, y_{2}, \ldots, y_{s}\right)=\sum_{j=1}^{s} \lambda_{i j} y_{j} .
$$

On setting $z_{j}=x_{j}^{k}$ for $1 \leq j \leq s$, we see that there is a real vector $\mathbf{z}=\left(z_{1}, \ldots, z_{s}\right) \in$ $\mathbb{R}^{s}$ such that $z_{j} \neq 0$ holds for $j \in J$, and we have

$$
L_{i}(\mathbf{z})=0 \quad \text { for } \quad 1 \leq i \leq R .
$$

Now, if $k$ is even, our choice of $\mathbf{z}$ ensures that we have $z_{j} \geq 0$ for $1 \leq j \leq s$. If $k$ is odd, then for each $j$, we may if necessary replace $z_{j}$ by $-z_{j}$, and replace the coefficients $\lambda_{i j}$ by $-\lambda_{i j}$ for $1 \leq i \leq R$, and consider the resulting system. In this manner, we may ensure that we have a solution $\mathbf{z}$ of (3.5) with $z_{j} \geq 0$ for $1 \leq j \leq s$ and $z_{j}>0$ for $j \in J$. Note that conditions (ii) and (iii) of Theorem 1.2 and conditions (3.2) and (3.3) are unaffected. Condition (iv) is also unaffected, since the sum $\sum_{x=1}^{q} e\left(\frac{x^{k}}{q} \sum_{i=1}^{r} \lambda_{i j} a_{i}\right)$ is always real for odd $k$ with $\lambda_{i j} \in \mathbb{Z}$, which may be seen by substituting $-x$ for $x$ in the sum.

Now suppose that $z_{j_{0}}=0$ for some $j_{0}$ satisfying $1 \leq j_{0} \leq s$. We clearly have $j_{0} \notin J$. Since we have assumed $\|\mathbf{F}\| \geq 1$, and thus certainly have $\|\mathbf{F}\| \neq 0$, we may fix a positive real number $\gamma$ with

$$
|\gamma| \leq \frac{\left|\operatorname{det}\left(A_{J}\right)\right| \min _{j \in J}\left|z_{j}\right|}{2 R^{R}\|\mathbf{F}\|^{R}}
$$

Since $A_{J}$ is nonsingular, there is a real vector $\mathbf{w}=\left(w_{1}, \ldots, w_{R}\right)$ such that we have

$$
A_{J} \mathbf{w}=-\gamma \boldsymbol{\lambda}_{j_{0}} .
$$

By Cramer's rule and Hadamard's rule, we certainly have

$$
\left|w_{i}\right| \leq \frac{R^{R}\|\mathbf{F}\|^{R} \gamma}{\left|\operatorname{det}\left(A_{J}\right)\right|} \leq \frac{1}{2} \min _{j \in J}\left|z_{j}\right| \quad \text { for } \quad 1 \leq i \leq R .
$$

Now define

$$
z_{j}^{\prime}=\left\{\begin{array}{cl}
z_{j_{i}}+w_{i} & \text { for } j=j_{i} \in J \\
\gamma & \text { for } j=j_{0} \\
z_{j} & \text { for } j \notin J \cup\left\{j_{0}\right\} .
\end{array}\right.
$$

Writing $\mathbf{z}^{\prime}=\left(z_{1}^{\prime}, z_{2}^{\prime}, \ldots, z_{s}^{\prime}\right)$, we have

$$
L_{i}\left(\mathbf{z}^{\prime}\right)=L_{i}(\mathbf{z})=0 \quad \text { for } \quad 1 \leq i \leq R .
$$

Also, we have $z_{j}^{\prime}>0$ for $j \in J \cup\left\{j_{0}\right\}$. All of the other components of $\mathbf{z}^{\prime}$ are equal to the respective components of $\mathbf{z}$; so we have replaced our real nonsingular solution by a real nonsingular solution that has one more positive component and that still satisfies the condition $z_{j}>0$ for $j \in J$. Repeating this process as many as $(s-R)$ times, we can find a nonsingular real solution $\mathbf{z}=\left(z_{1}, \ldots, z_{s}\right)$ with $z_{j}>0$ for $1 \leq j \leq s$.

Thus, scaling if necessary, we may choose a real number $\delta$ and real numbers $z_{1}, z_{2}, \ldots, z_{s}$ that satisfy

$$
\begin{aligned}
0<\delta \leq z_{j} \leq \frac{1}{2} \quad \text { for } \quad 1 \leq j \leq s, \quad \text { and } \\
L_{i}(\mathbf{z})=0 \quad \text { for } \quad 1 \leq i \leq R .
\end{aligned}
$$


To sum up, in this section, we have demonstrated that to prove Theorem 1.2 it is enough to consider a system of forms $F_{1}, F_{2}, \ldots, F_{R}$ as in Theorem 1.2, with the added assumptions that $\|\mathbf{F}\| \geq 1$ holds, that the coefficient matrix $A$ of the system satisfies the conditions (3.2) and (3.3), and that there is a real vector $\mathbf{z}$ satisfying (3.6). In sections 4-9, we prove Theorem 1.2 under these additional assumptions.

\section{The Davenport-Heilbronn Method: The Setup}

Now we proceed with the proof of Theorem 1.2 under the additional assumptions we made above. We shall essentially use the Hardy-Littlewood method, in an involved form. We combine the methods of Bentkus and Götze [3] with those of Nadesalingam and Pitman [16].

We note that throughout the paper, implicit constants in the notation $o()$ and $O()$ and $\ll$ and $\gg$ may depend on $R, s, k, \delta, \ell$, the coefficients of the forms $F_{1}, \ldots$, $F_{R}$, and the real vector $\mathbf{z}$.

We consider the number of solutions of the system

$$
\begin{array}{rlrr}
F_{i}(\mathbf{y})=0 & \text { for } & 1 \leq i \leq r, \\
\left|F_{i}(\mathbf{y})\right|<1 & \text { for } & r+1 \leq i \leq R .
\end{array}
$$

In the usual fashion, we use a real-valued, even kernel function $K: \mathbb{R} \rightarrow \mathbb{R}$ to give a lower bound for the number of integral solutions of the system (4.1) in a certain range. Define such a function $K$, for any real number $\alpha$, by

$$
K(\alpha)=\left(\frac{\sin \pi \alpha}{\pi \alpha}\right)^{2} .
$$

By Lemma 14.1 of [2], for any real number $u$, the function $K$ satisfies the identity

$$
\psi(u)=\int_{-\infty}^{\infty} e(\beta u) K(\beta) d \beta=\left\{\begin{array}{cll}
0 & \text { if } & |u| \geq 1 \\
1-|u| & \text { if } & |u|<1 .
\end{array}\right.
$$

The function $K$ satisfies, for real numbers $\beta$, the bound

$$
|K(\beta)| \ll \min \left(1,|\beta|^{-2}\right) .
$$

We will also use the identity

$$
\int_{0}^{1} e(\alpha n) d \alpha=\left\{\begin{array}{lll}
1 & \text { if } & n=0 \\
0 & \text { if } & n \in \mathbb{Z} \backslash\{0\} .
\end{array}\right.
$$

Now for positive real numbers $P$ and $Q$ satisfying $Q \leq P$, we define the so-called $Q$-smooth numbers to be the set

$$
\mathcal{A}(P, Q)=\{x \in \mathbb{Z} \text { with } 1 \leq x \leq P \text { such that } p \mid x \Longrightarrow p \leq Q\} .
$$

Fix a positive real number $\eta$, to be chosen later, so that it will satisfy the requirements of Lemmas 5.5 and 6.1 Then for real numbers $\alpha$ and $P$ with $P \geq 1$, we define the exponential sum $g(\alpha)$ over the smooth numbers by

$$
g(\alpha)=g(\alpha, P)=\sum_{x \in \mathcal{A}\left(P, P^{\eta}\right)} e\left(\alpha x^{k}\right) .
$$

Also, for $1 \leq j \leq s$, and real vectors $\boldsymbol{\alpha} \in \mathbb{R}^{R}$, we define the linear forms

$$
\Lambda_{j}(\boldsymbol{\alpha})=\sum_{i=1}^{R} \lambda_{i j} \alpha_{i} \quad \text { and } \quad \Lambda_{j}^{(r)}(\boldsymbol{\alpha})=\sum_{i=1}^{r} \lambda_{i j} \alpha_{i} .
$$


Then we also define, for $\boldsymbol{\alpha} \in \mathbb{R}^{R}$ and real numbers $P$ with $P \geq 1$, and for $1 \leq j \leq s$, the functions

$$
g_{j}(\boldsymbol{\alpha})=g_{j}(\boldsymbol{\alpha}, P)=g\left(\Lambda_{j}(\boldsymbol{\alpha}), P\right) .
$$

We define as well

$$
W=[0,1]^{r} \times \mathbb{R}^{R-r} .
$$

Now let $\mathcal{N}(P)$ be the number of solutions of the system (4.1) with $x_{j} \in \mathcal{A}\left(P, P^{\eta}\right)$ for $1 \leq j \leq s$. By using the property (4.3) of the function $K(\alpha)$ and the identity (4.5), one can see that we have

$$
\mathcal{N}(P) \geq \sum_{\substack{x_{j} \in \mathcal{A}\left(P, P^{\eta}\right) \\ 1 \leq j \leq s}} \int_{W} e\left(\sum_{i=1}^{R} \alpha_{i} F_{i}(\mathbf{x})\right)\left(\prod_{i=r+1}^{R} K\left(\alpha_{i}\right)\right) d \boldsymbol{\alpha} ;
$$

observe that this last remark is justified by the fact that the integral converges absolutely, which follows from (4.4), whence we may write the integral as a product of $R$ integrals. By pulling the sums into the integral, we may rewrite the above bound in the form

$$
\mathcal{N}(P) \geq \int_{W} \prod_{j=1}^{s} g_{j}(\boldsymbol{\alpha}) \prod_{i=r+1}^{R} K\left(\alpha_{i}\right) d \boldsymbol{\alpha} .
$$

Thus, to prove Theorem 1.2 it is enough to show that the right-hand side of (4.9) is at least 2 .

To this end, we give a dissection of the region of integration $W$ into three subsets. Roughly speaking, we expect that the main contribution to the integral in (4.9) comes from the region where the first $r$ components of $\boldsymbol{\alpha}$ are "close" to rational numbers with small denominators and the last $R-r$ components of $\boldsymbol{\alpha}$ are very small in absolute value. We will show that the contribution to the integral in (4.9) from this region, the so-called major arcs, is positive and "large", and we will also show that the contribution to the integral from the other regions is smaller, and thus the integral over all of $W$ is positive.

For notational ease, we set

$$
B=\frac{1}{4(R+1)}
$$

We now define, for positive integers $q$ and integral vectors $\mathbf{a}=\left(a_{1}, a_{2}, \ldots, a_{r}\right) \in$ $\mathbb{Z}^{r}$, and real numbers $P$ with $P \geq 2$, the region $\mathcal{M}(q, \mathbf{a})$, or $\mathcal{M}(q, \mathbf{a}, P)$, by

$$
\begin{array}{r}
\mathcal{M}(q, \mathbf{a})=\left\{\boldsymbol{\alpha} \in[0,1]^{r} \times\left[-(\log P)^{B} P^{-k},(\log P)^{B} P^{-k}\right]^{R-r}:\right. \\
\left.\left\|\alpha_{i}-\frac{a_{i}}{q}\right\| \leq(\log P)^{B} P^{-k} \text { for } 1 \leq i \leq r\right\} ;
\end{array}
$$

here $\|x\|$ denotes the distance from the real number $x$ to the nearest integer. We define the major arcs to be the region

$$
\mathcal{M}=\mathcal{M}(P)=\bigcup_{1 \leq q \leq(\log P)^{B}} \bigcup_{\substack{\mathbf{a}(\bmod q) \\\left(a_{1}, \ldots, a_{r}, q\right)=1}} \mathcal{M}(q, \mathbf{a}, P),
$$


where by a $(\bmod q)$ we mean that a runs over vectors $\mathbf{a} \in \mathbb{Z}^{r}$ such that one has $1 \leq a_{i} \leq q$ for $1 \leq i \leq r$.

In section 5, we will prove the existence of a function $T(P)$, with $T(P) \geq 1$ and $\lim _{P \rightarrow \infty} T(P)=\infty$, and satisfying a certain property. The function will depend on $B$ and the coefficients of the forms $F_{i}$. We define the minor arcs to be the region

$$
\mathfrak{m}=\mathfrak{m}(P)=\left([0,1]^{r} \times[-T(P), T(P)]^{R-r}\right) \backslash \mathcal{M}(P) .
$$

Finally, we define the trivial arcs to be the set

$$
\mathfrak{t}=\mathfrak{t}(P)=\{\boldsymbol{\alpha} \in W:|\boldsymbol{\alpha}|>T(P)\} .
$$

\section{An Analogue of Weyl's Inequality}

In this section, we give an analogue of Weyl's inequality. For any real number $T$ with $T \geq 1$, define the region

$$
\mathfrak{m}_{T}=\mathfrak{m}_{T}(P)=\left([0,1]^{r} \times[-T, T]^{R-r}\right) \backslash \mathcal{M}(P) .
$$

We now state the central lemma of this section.

Lemma 5.1. Fix a positive real number $T$ with $T \geq 1$. Define the forms $F_{i}(\mathbf{x})$ as in (1.2) for $1 \leq i \leq R$, the region $\mathfrak{m}_{T}(P)$ as above, and $g_{j}(\boldsymbol{\alpha}, P)$ for $1 \leq j \leq s$ as in (4.8). Suppose that the coefficient matrix $A$ associated with the system $\mathbf{F}$ has rank $R$. Suppose also that the irrationality condition (iii) of Theorem 1.2 holds. Then one has

$$
\lim _{P \rightarrow \infty} \sup _{\boldsymbol{\alpha} \in \mathfrak{m}_{T}(P)} \frac{\prod_{j=1}^{s}\left|g_{j}(\boldsymbol{\alpha}, P)\right|}{P^{s}}=0 .
$$

Observe that trivially one has $\prod_{j=1}^{s}\left|g_{j}(\boldsymbol{\alpha}, P)\right| \leq P^{s}$; so we are only seeking a slight improvement over the trivial bound. We also note that the central ideas of the proof stem from the work of Bentkus and Götze [3].

In order to prove Lemma 5.1, we first need to give another lemma, which is essentially a combination of two analogues of Weyl's inequality for exponential sums over smooth numbers. We first quote these two analogues, essentially due to Vaughan and Wooley, as they are presented in [5] as Lemmas 3 and 4, respectively.

Lemma 5.2. Let $\alpha$ and $P$ be real numbers with $P \geq 2$. Define $g(\alpha)=g(\alpha, P)$ as in (4.6). Fix a positive real number $\epsilon$. Then for sufficiently small $\eta$, there is a positive real number $\gamma$ that depends only on $k$ such that either one has $|g(\alpha, P)| \leq P^{1-\gamma}$, or there are relatively prime integers $a$ and $q$ with $q \geq 1$ that satisfy

$$
g(\alpha, P) \ll q^{\epsilon} P\left(q+P^{k}|q \alpha-a|\right)^{-1 /(2 k)}(\log P)^{3} .
$$

Lemma 5.3. Let $\alpha$ and $P$ be real numbers with $P \geq 3$. Define $g(\alpha)=g(\alpha, P)$ as in (4.6) with $0<\eta<1 / 2$. Fix positive real numbers $A$ and $\epsilon$. Suppose that a and $q$ are relatively prime integers with $1 \leq q \leq(\log P)^{A}$ and $|q \alpha-a| \leq(\log P)^{A} P^{-k}$. Then one has

$$
g(\alpha, P) \ll_{A, \epsilon} q^{\epsilon} P\left(q+P^{k}|q \alpha-a|\right)^{-1 / k} .
$$


We now state the combination of these lemmas.

Lemma 5.4. Define $\gamma=\gamma(k)$ as in Lemma 5.2. Fix positive real numbers $\theta$ and $B^{\prime}$. Suppose that $P$ is a real number with $P \geq 3$, and that $\mu$ is a real number with

$$
\mu>\max \left((\log P)^{-B^{\prime}}, P^{-\gamma}\right) .
$$

Define $g(\alpha)=g(\alpha, P)$ as in (4.6), with $\eta$ sufficiently small, and suppose that one has

$$
|g(\alpha, P)| \geq \mu P .
$$

Then there exists a positive integer $q$ and an integer $a$ with $(a, q)=1$ and

$$
q \ll_{B^{\prime}, k, \theta} \mu^{-k-k \theta} \quad \text { and } \quad|q \alpha-a| \ll_{B^{\prime}, k, \theta} \mu^{-k-k \theta} P^{-k} .
$$

Proof. It is clearly enough to assume that we have $\theta \leq 1 / 2$. We apply Lemma 5.2 with the choice $\epsilon=\theta /(2 k)$. By (5.3) and (5.4), there exist relatively prime integers $a$ and $q$ with $q \geq 1$ such that one has

$$
\mu P \leq|g(\alpha, P)| \ll q^{\theta /(2 k)} P\left(q+P^{k}|q \alpha-a|\right)^{-1 /(2 k)}(\log P)^{3} .
$$

It follows that

$$
q^{1-\theta} \ll \mu^{-2 k}(\log P)^{6 k} \quad \text { and } \quad P^{k}|q \alpha-a| \ll q^{\theta} \mu^{-2 k}(\log P)^{6 k} .
$$

By (5.3) and the condition $\theta \leq 1 / 2$, we certainly have

$$
q \ll(\log P)^{\frac{2 k\left(B^{\prime}+3\right)}{1-\theta}} \text { and }|q \alpha-a| \ll(\log P)^{\frac{4 k\left(B^{\prime}+3\right)}{1-\theta}} P^{-k} .
$$

Now we may apply Lemma [5.3, for large $P$, choosing $A=5 k\left(B^{\prime}+3\right) /(1-\theta)$, say, and $\epsilon=\theta /(2 k)$. We obtain

$$
\mu \ll q^{\theta /(2 k)}\left(q+P^{k}|q \alpha-a|\right)^{-1 / k} .
$$

It follows that one has

$$
q \ll \mu^{-k} q^{\theta / 2} \quad \text { and } \quad P^{k}|q \alpha-a| \ll \mu^{-k} q^{\theta / 2} .
$$

Thus, since $\mu \leq 1$ must hold, we have

$$
q \ll \mu^{-\frac{2 k}{2-\theta}} \ll \mu^{-k-k \theta} \quad \text { and } \quad|q \alpha-a| \ll \mu^{-k+\frac{\theta}{2}(-k-k \theta)} P^{-k} \ll \mu^{-k-k \theta} P^{-k} \text {. }
$$

Thus the proof of Lemma 5.4 is complete.

Now we are able to give the proof of Lemma 5.1.

Proof. Suppose for the sake of contradiction that the condition (5.2) does not hold. Then there exist a positive real number $\epsilon$, an increasing sequence of positive real numbers $P_{n}$ with $\lim _{n \rightarrow \infty} P_{n}=\infty$, and a sequence of real vectors $\boldsymbol{\alpha}_{n} \in \mathfrak{m}_{T}\left(P_{n}\right)$ with

$$
\prod_{j=1}^{s}\left|g_{j}\left(\boldsymbol{\alpha}_{n}, P_{n}\right)\right|>\epsilon P_{n}^{s} .
$$

We may clearly assume that we have $\epsilon<1$. By trivial estimates, we have

$$
\left|g_{j}\left(\boldsymbol{\alpha}_{n}, P_{n}\right)\right|>\epsilon P_{n} \quad \text { for } \quad 1 \leq j \leq s .
$$

Now we apply Lemma 5.4 to the sums $g_{j}\left(\boldsymbol{\alpha}_{n}, P_{n}\right)=g\left(\Lambda_{j}\left(\boldsymbol{\alpha}_{n}\right), P_{n}\right)$ for all sufficiently large choices of $n$. For sufficiently large $n$, we have the bounds $\epsilon \geq P_{n}^{-\gamma}$ and 
$\epsilon>\left(\log P_{n}\right)^{-1}$, and also $P_{n} \geq 3$. Thus we may apply Lemma 5.4 with $\mu=\epsilon$ and $\theta=1 / k$ and $B^{\prime}=1$. Therefore, there are constants $c_{1}$ and $c_{2}$ that depend only on $k$ such that for large $n$ and for $1 \leq j \leq s$, there are integers $q_{n j}$ and $a_{n j}$ that satisfy

$$
1 \leq q_{n j} \leq c_{1} \epsilon^{-k-1} \quad \text { and } \quad\left|\Lambda_{j}(\boldsymbol{\alpha}) q_{n j}-a_{n j}\right| \leq c_{2} \epsilon^{-k-1} P_{n}^{-k} .
$$

It follows from these bounds and the definition (5.1) of $\mathfrak{m}_{T}\left(P_{n}\right)$ that we have

$$
\left|a_{n j}\right| \leq c_{2} \epsilon^{-k-1} P_{n}^{-k}+c_{1} \epsilon^{-k-1} R T\|\mathbf{F}\|
$$

for all $j$ with $1 \leq j \leq s$, and all large $n$.

For fixed $\epsilon$ and $T$, we thus have that $\left|a_{n j}\right|$ and $q_{n j}$ are uniformly bounded. So there are only finitely many possible $(2 s)$-tuples

$$
\left(q_{n 1}, q_{n 2}, \ldots, q_{n s}, a_{n 1}, a_{n 2}, \ldots, a_{n s}\right) .
$$

Therefore one such $(2 s)$-tuple, say $\left(q_{1}, \ldots, q_{s}, a_{1}, \ldots, a_{s}\right)$, occurs infinitely often. Thus there is some subsequence, say $\left\{\tilde{n}_{m}\right\}$, with

$$
\left(q_{\tilde{n}_{m} 1}, \ldots, q_{\tilde{n}_{m} s}, a_{\tilde{n}_{m} 1}, \ldots, a_{\tilde{n}_{m} s}\right)=\left(q_{1}, \ldots, q_{s}, a_{1}, \ldots, a_{s}\right)
$$

for all $m \in \mathbb{Z}^{+}$.

Since the sequence $\left\{\boldsymbol{\alpha}_{\tilde{n}_{m}}\right\}$ is contained within the compact set $[0,1]^{r} \times$ $[-T, T]^{R-r}$, there is a further subsequence $\left\{\boldsymbol{\alpha}_{n_{m}}\right\}$ and a vector $\boldsymbol{\alpha}_{0} \in[0,1]^{r} \times$ $[-T, T]^{R-r}$ such that

$$
\lim _{m \rightarrow \infty} \boldsymbol{\alpha}_{n_{m}}=\boldsymbol{\alpha}_{0}
$$

Our goal in the remainder of the lemma is to show that for sufficiently large values of $m$, we have $\boldsymbol{\alpha}_{n_{m}} \in \mathcal{M}\left(P_{n_{m}}\right)$, which contradicts our original assumption.

By (5.5) and the defining property of the subsequence $\left\{\tilde{n}_{m}\right\}$, we have

$$
\left|\Lambda_{j}\left(\boldsymbol{\alpha}_{n_{m}}\right) q_{j}-a_{j}\right| \leq c_{2} \epsilon^{-k-1} P_{n_{m}}^{-k} \quad \text { for } \quad 1 \leq j \leq s \text { and for all } m \in \mathbb{Z}^{+} \text {. }
$$

Taking the limit of both sides of (5.6) as $m$ goes to infinity, we obtain

$$
\Lambda_{j}\left(\boldsymbol{\alpha}_{0}\right)=\frac{a_{j}}{q_{j}} \quad \text { for } \quad 1 \leq j \leq s .
$$

Because condition (iii) of Theorem 1.2 holds, denoting $\boldsymbol{\alpha}_{0}=\left(\alpha_{01}, \alpha_{02}, \ldots, \alpha_{0 R}\right)$ we must have

$$
\alpha_{0(r+1)}=\alpha_{0(r+2)}=\cdots=\alpha_{0 R} .
$$

Therefore we have

$$
\Lambda_{j}^{(r)}\left(\boldsymbol{\alpha}_{0}\right)=\frac{a_{j}}{q_{j}} \quad \text { for } \quad 1 \leq j \leq s .
$$

Now, by (5.6) and (5.7), we have

$$
\left|\Lambda_{j}\left(\boldsymbol{\alpha}_{n_{m}}-\boldsymbol{\alpha}_{0}\right)\right|=\left|\Lambda_{j}\left(\boldsymbol{\alpha}_{n_{m}}\right)-\frac{a_{j}}{q_{j}}+\frac{a_{j}}{q_{j}}-\Lambda_{j}\left(\boldsymbol{\alpha}_{0}\right)\right| \leq c_{2} \epsilon^{-k-1} P_{n_{m}}^{-k}
$$

for $1 \leq j \leq s$ and for all $m \in \mathbb{Z}^{+}$. Now, because $A$ has full rank, we may assume by relabeling variables if necessary that the submatrix $A_{1}$, defined as in (3.2), is nonsingular. Because of this and because the bound (5.9) holds, in particular, for $1 \leq j \leq R$, we must have $\left|\boldsymbol{\alpha}_{n_{m}}-\boldsymbol{\alpha}_{0}\right| \leq c_{3}(\mathbf{F}) \epsilon^{-k-1} P_{n_{m}}^{-k}$ for some constant $c_{3}=c_{3}(\mathbf{F})$ and for all $m \in \mathbb{Z}^{+}$. Therefore, by (5.8), we must have

$$
\boldsymbol{\alpha}_{n_{m}} \in[0,1]^{r} \times\left[-c_{3} \epsilon^{-k-1} P_{n_{m}}^{-k}, c_{3} \epsilon^{-k-1} P_{n_{m}}^{-k}\right]^{R-r} \quad \text { for } \quad m \in \mathbb{Z}^{+} .
$$


If $r=0$ holds, then for $m$ sufficiently large, one must have $\boldsymbol{\alpha}_{n_{m}} \in \mathcal{M}\left(P_{n_{m}}\right)$. But this contradicts our original assumption that the sequence $\boldsymbol{\alpha}_{n}$ satisfies $\boldsymbol{\alpha}_{n} \in \mathfrak{m}_{T}\left(P_{n}\right)$, whence the equality (5.2) must hold.

So we may assume for the remainder of the proof that $0<r \leq R$ holds. Then, using (5.6) and (5.10), for $m \in \mathbb{Z}^{+}$and for $1 \leq j \leq s$, we have

$$
\left|\Lambda_{j}^{(r)}\left(\boldsymbol{\alpha}_{n_{m}}\right)-\frac{a_{j}}{q_{j}}\right|=\left|\Lambda_{j}\left(\boldsymbol{\alpha}_{n_{m}}\right)-\frac{a_{j}}{q_{j}}-\sum_{i=r+1}^{R} \lambda_{i j} \alpha_{n_{m} i}\right| \ll \epsilon^{-k-1} P_{n_{m}}^{-k}
$$

Since $A_{1}$ is nonsingular, there is an $r \times r$ submatrix, say $A_{0}$, of $A_{1}$ that is nonsingular. We assume for ease of notation that $A_{0}$ is the upper left-hand $r \times r$ submatrix of $A_{1}$, noting that the other cases all follow in the same fashion as this case. For any real vector $\boldsymbol{\alpha}=\left(\alpha_{1}, \ldots, \alpha_{R}\right)$, write $\boldsymbol{\alpha}^{\prime}=\left(\alpha_{1}, \ldots, \alpha_{r}\right)$. By (5.11), we have

$$
A_{0}^{T} \boldsymbol{\alpha}_{n_{m}}^{\prime}=\left[\begin{array}{c}
a_{1} / q_{1} \\
a_{2} / q_{2} \\
\vdots \\
a_{r} / q_{r}
\end{array}\right]+\left[\begin{array}{c}
w_{m 1} \\
w_{m 2} \\
\vdots \\
w_{m r}
\end{array}\right],
$$

for some real vector $\mathbf{w}_{m}=\left(w_{m 1}, \ldots, w_{m r}\right)$ with $\left|\mathbf{w}_{m}\right| \ll \epsilon^{-k-1} P_{n_{m}}^{-k}$. Since we have assumed that $A_{0}$ is nonsingular, we may use Cramer's rule to find $\mathbf{b}=\left(b_{1}, \ldots, b_{r}\right)$ with

$$
A_{0}^{T} \mathbf{b}=\left[\begin{array}{c}
a_{1} / q_{1} \\
a_{2} / q_{2} \\
\vdots \\
a_{r} / q_{r}
\end{array}\right] .
$$

Since $A_{0}^{T}$ has integral entries, one may see that $b_{i}$ has the form $b_{i}=d_{i} / q$ for $1 \leq i \leq r$, where $d_{i}$ is an integer, and $q$ is a positive integer that satisfies

$$
q \leq\left(q_{1} q_{2} \cdots q_{r}\right) \operatorname{det}\left(A_{0}^{T}\right) \leq c_{4}(\mathbf{F}) \epsilon^{-r(k+1)},
$$

where the last bound follows from (5.5).

We may assume, by reducing if necessary, that we have $\left(d_{1}, d_{2}, \ldots, d_{r}, q\right)=1$. By Cramer's rule again, we may find $\mathbf{v}_{m} \in \mathbb{R}^{r}$ with $A_{0}^{T} \mathbf{v}_{m}=\mathbf{w}_{m}$, where we have

$$
\left|\mathbf{v}_{m}\right| \leq c_{5}(\mathbf{F}) \epsilon^{-k-1} P_{n_{m}}^{-k}
$$

Write $\mathbf{d}=\left(d_{1}, \ldots, d_{r}\right)$, and if $d_{i}=0$ for some $i$, define $d_{i}$ to be $q$ instead. Then we have

$$
\boldsymbol{\alpha}_{n_{m}}^{\prime} \equiv \frac{1}{q} \mathbf{d}+\mathbf{v}_{m} \quad(\bmod 1) \quad \text { for } \quad m \in \mathbb{Z}^{+} .
$$

Now fix any choice of $m$ large enough so that we have

$$
\left(\log P_{n_{m}}\right)^{B} \geq \max \left(c_{3}(\mathbf{F}), c_{4}(\mathbf{F}), c_{5}(\mathbf{F})\right) \epsilon^{-r(k+1)} .
$$

Then by (5.10) we have

$$
\boldsymbol{\alpha}_{n_{m}} \in[0,1]^{r} \times\left[-\left(\log P_{n_{m}}\right)^{B} P_{n_{m}}^{-k},\left(\log P_{n_{m}}\right)^{B} P_{n_{m}}^{-k}\right]^{R-r}
$$

for this choice of $m$. Now write $\hat{\mathbf{d}}=\left(d_{1}, d_{2}, \ldots, d_{r}, 0,0, \ldots, 0\right)$, where there are $R-r$ zeros here, and define $\hat{\mathbf{v}}_{m}$ similarly. Then, setting

$$
\mathbf{u}=\hat{\mathbf{v}}_{m}+\left(0,0, \ldots, 0, \alpha_{n_{m}(r+1)}, \ldots, \alpha_{n_{m} R}\right),
$$

we have

$$
\boldsymbol{\alpha}_{n_{m}} \equiv \frac{1}{q} \hat{\mathbf{d}}+\mathbf{u} \quad(\bmod 1),
$$


where $\left(d_{1}, d_{2}, \ldots, d_{r}, q\right)=1$ and where, by combining (5.14) with (5.10), (5.12) and (5.13), we have

$$
1 \leq q \leq\left(\log P_{n_{m}}\right)^{B} \quad \text { and } \quad|\mathbf{u}| \leq\left(\log P_{n_{m}}\right)^{B} P_{n_{m}}^{-k} .
$$

Thus, recalling definition (4.11), we have $\boldsymbol{\alpha}_{n_{m}} \in \mathcal{M}\left(q, \mathbf{d}, P_{n_{m}}\right)$ for our particular choice of $m$ and, in fact, recalling (4.12), we also have $\boldsymbol{\alpha}_{n_{m}} \in \mathcal{M}\left(P_{n_{m}}\right)$. As in the case $r=0$, this is a contradiction, whence the equality (5.2) must in fact hold. This completes the proof of Lemma 5.1

At this point, we make an observation about the lemma for those familiar with earlier arguments of this type. We note that in previous work by the author ([10], [11]), the analogue of our Lemma 5.1 was proved with two different methods, for two subregions of the region $\mathfrak{m}_{T}(P)$. If we were to proceed by analogy with earlier arguments, we would instead have to treat a region $\mathfrak{m}_{T, T_{0}}(P)$, say, in place of $\mathfrak{m}_{T}(P)$, for positive real numbers $T_{0}$ with $T_{0} \leq T$. The new region would be defined by

$$
\mathfrak{m}_{T, T_{0}}(P)=\mathfrak{m}_{T}(P) \cap\left\{\boldsymbol{\alpha}:|\boldsymbol{\alpha}| \geq T_{0}\right\} .
$$

Essentially by combining the arguments used for each region in previous proofs, we are able to dispense with the requirement $|\boldsymbol{\alpha}| \geq T_{0}$.

Having done most of the work, we can now give a lemma that essentially says that $\prod_{j=1}^{s}\left|g_{j}(\boldsymbol{\alpha}, P)\right|$ is small for $\boldsymbol{\alpha} \in \mathfrak{m}$. The idea of using such a lemma is due originally to Bentkus and Götze [3].

Lemma 5.5. Define the forms $F_{i}(\mathbf{x})$ as in (1.2) for $1 \leq i \leq R$ and the exponential sums $g_{j}(\boldsymbol{\alpha}, P)$ for $1 \leq j \leq s$ as in (4.8), with $\eta$ sufficiently small. Suppose that the coefficient matrix $A$ associated with the system $\mathbf{F}$ has rank $R$. Suppose also that the irrationality condition (iii) of Theorem 1.2 holds. Then there exists a function $T(P)$ that depends only on $B, \eta$ and the coefficients of the forms $F_{1}, F_{2}, \ldots, F_{R}$, that satisfies $T(P) \geq 1$ and

$$
\lim _{P \rightarrow \infty} T(P)=\infty
$$

and such that if we define $\mathfrak{m}(P)$ as in (4.13) with this choice of $T(P)$, then one has

$$
\sup _{\boldsymbol{\alpha} \in \mathfrak{m}(P)} \prod_{j=1}^{s}\left|g_{j}(\boldsymbol{\alpha}, P)\right|=o\left(P^{s}\right) .
$$

Proof. The lemma is very similar to Lemma 6 of [10] and Lemma 4 of [11, and the proof follows in a similar fashion.

We note that this lemma (and Lemma 5.19) holds for any positive choice of $B$, but that the function above that is $o\left(P^{s}\right)$ depends on $B$. We have stated this lemma in a general fashion in the hopes that it may be useful for future workers.

We observe that one could ensure that the function that is $o\left(P^{s}\right)$ depends only on $B, \eta$ and $2 R-r$ of the coefficients. This follows, with some effort, after finding a subset $J \subseteq\{1,2, \ldots, s\}$ with $|J|=2 R-r$ such that the conditions $\Lambda_{j}(\boldsymbol{\alpha}) \in \mathbb{Q}$ for $j \in J$, taken together, imply that $\alpha_{r+1}=\cdots=\alpha_{R}=0$. This can be proved, although our method of proof, at least, is not straightforward. 
In the remainder of the paper, we fix a function $T(P)$ that satisfies the conclusions of the above lemma. We note that this is the special function we referred to above in section 4 and is used to define the minor arcs and trivial arcs.

We observe at this point that we could obtain corresponding results which are very similar to Lemmas 5.1 and 5.5 if the exponential sums $g_{j}$ were replaced by exponential sums over a complete interval. The only major change needed would be to use Lemma 2 of [11] in place of our Lemma 5.4.

\section{The Minor Arcs}

In this section, our goal is to show that the contribution from the minor arcs to the integral in (4.9) is $o\left(P^{s-R k}\right)$. We first give a lemma, which is essentially a restatement of results due to Vaughan [19], 20], and results due to Wooley [23].

Lemma 6.1. Suppose that $k$ is an integer with $k \geq 2$. Define $g(\alpha)$ as in (4.6), with $\eta$ sufficiently small. Then there is an absolute positive constant $C^{\prime}$ such that if $t$ is a real number satisfying either

$$
\text { (i) } \quad t \geq \min \left(2^{k}, k(\log k+\log \log k+2)+\frac{C^{\prime} k \log \log k}{\log k}\right) \quad \text { for } \quad k \geq 3 \text {, }
$$

or

$$
\text { (ii) } t>4 \text { for } k=2 \text {, }
$$

then one has

$$
\int_{0}^{1}|g(\alpha)|^{t} \ll_{\eta} P^{t-k}
$$

We observe that one could certainly improve on the lemma in certain cases, but we choose to use only the above bounds for our results.

Proof. If the first bound of condition (i) holds, then the result is Lemma 6 of [11, which is essentially due to Vaughan [19], 20]. If on the other hand, the second bound of condition (i) holds, then we may essentially quote Lemma 7 of [11], which itself follows almost immediately from work of Wooley [23]. We note that the 3 in Lemma 7 of [11] has been replaced by a 2 here; I am grateful to Scott Parsell for showing me the technique one uses to make this improvement.

In the case in which (ii) holds, we give a proof for completeness. Define

$$
\epsilon=t-4 \text {. }
$$

We need only prove that one has

$$
\int_{0}^{1}|g(\alpha)|^{4+\epsilon} d \alpha \ll P^{2+\epsilon}
$$

Clearly, we may assume that $\epsilon \leq 1$ holds. For convenience, we write

$$
G=\frac{2}{\epsilon}
$$

Define

$$
\mathfrak{N}=\left\{\alpha \in[0,1]:|g(\alpha)|>P(\log P)^{-G}\right\} .
$$

Also, for positive integers $m$, define

$$
\mathfrak{N}_{m}=\left\{\alpha \in \mathfrak{N}: 2^{-m-1} P \leq|g(\alpha)| \leq 2^{-m} P\right\} .
$$


Now for $\alpha \in \mathfrak{N}_{m}$, we apply Lemma 5.4 with the choice $B^{\prime}=G$. Thus, for large $P$ and any positive real number $\delta$, there exist coprime integers $a$ and $q$ with $q \geq 1$, and

$$
q \ll 2^{m(2+\delta)} \quad \text { and } \quad\left|\alpha-\frac{a}{q}\right| \ll q^{-1} 2^{m(2+\delta)} P^{-2} .
$$

Thus we have

$$
\int_{\mathfrak{N}_{m}}|g(\alpha)|^{4+\epsilon} d \alpha \ll \sum_{q \ll 2^{m(2+\delta)}} \sum_{a=1}^{q}\left(2^{-m} P\right)^{4+\epsilon} q^{-1} 2^{m(2+\delta)} P^{-2} \ll P^{2+\epsilon} 2^{-m(\epsilon-2 \delta)} .
$$

It follows for $\delta<\epsilon / 2$ that

$$
\int_{\mathfrak{N}}|g(\alpha)|^{4+\epsilon} d \alpha \ll P^{2+\epsilon} \sum_{m=0}^{\infty} 2^{-m(\epsilon-2 \delta)} \ll P^{2+\epsilon} .
$$

On the other hand, one has

$$
\int_{[0,1] \backslash \mathfrak{N}}|g(\alpha)|^{4+\epsilon} d \alpha \ll\left(\sup _{\alpha \in[0,1] \backslash \mathfrak{N}}|g(\alpha)|^{\epsilon}\right) \int_{[0,1]}|g(\alpha)|^{4} d \alpha .
$$

But $\int_{[0,1]}|g(\alpha)|^{4} d \alpha$ is less than or equal to the number of solutions of the equation

$$
x_{1}^{2}+x_{2}^{2}=x_{3}^{2}+x_{4}^{2}
$$

with $1 \leq x_{i} \leq P$ for $1 \leq i \leq 4$. This is bounded by a constant multiple of $P^{2} \log P$, a well-known result, which can be proved by elementary means. Thus from (6.3) and the definition of $\mathfrak{N}$, we have

$$
\int_{[0,1] \backslash \mathfrak{N}}|g(\alpha)|^{4+\epsilon} d \alpha \ll\left(P(\log P)^{-G}\right)^{\epsilon} P^{2} \log P \ll P^{2+\epsilon}(\log P)^{-1},
$$

by our choice of $G$. Combining this bound with (6.2) completes the proof of Lemma 6.1 .

We note that (6.1) is an example of what one might call an "exact Hua inequality". In most work using the Hardy-Littlewood method, one uses bounds of the type (6.1) where one only needs to show that for any $\epsilon>0$, the left side of (6.1) can be bounded by $P^{t-k+\epsilon}$. Dispensing with this $\epsilon$ is crucial for our work. The use of such an inequality stems from the work of Bentkus and Götze [3].

For the remainder of the paper, we now fix a choice of $\eta$ so that Lemmas 5.5 and 6.1 hold for this choice. Now we turn to what is essentially our analogue of Hua's inequality. It is very similar to Lemma 8 of [10].

Lemma 6.2. There is an absolute positive real constant $\tilde{C}_{2}$ with the following property:

Assume that the forms $F_{1}, F_{2}, \ldots, F_{R}$ are as in Theorem 1.2, with coefficient matrix A satisfying (3.2) and (3.3). Assume that $\ell$ is a positive integer satisfying

$$
\begin{array}{cl}
\ell \geq 5 & \text { for } k=2, \text { and } \\
\ell \geq \min \left(2^{k}+1, k(\log k+\log \log k+2)+\frac{\tilde{C}_{2} k \log \log k}{\log k}\right) & \text { for } \quad k \geq 3 .
\end{array}
$$


Define the exponential sums $g_{j}(\boldsymbol{\alpha}, P)$ as in (4.8), and define the function $K$ as in (4.2). Let $d(P)$ be a nonnegative real-valued function, and let $\mathfrak{n}$ be any subset of the region

$$
\mathfrak{d}=\{\boldsymbol{\alpha} \in W \text { with }|\boldsymbol{\alpha}| \geq d(P)\}
$$

Also, define

$$
h(\mathfrak{n}, P)=\sup _{\alpha \in \mathfrak{n}} P^{-s} \prod_{j=1}^{s}\left|g_{j}(\boldsymbol{\alpha}, P)\right| .
$$

Then there is a positive real number $\nu$ that depends only on $k$ such that one has

$$
\int_{\mathfrak{n}} \prod_{j=1}^{s}\left|g_{j}(\boldsymbol{\alpha}, P)\right| \prod_{i=r+1}^{R}\left|K\left(\alpha_{i}\right)\right| d \boldsymbol{\alpha} \ll(h(\mathfrak{n}, P))^{\nu} \min \left(1, d(P)^{-1}\right) P^{s-R k} .
$$

Proof. Observe first that for any real number $\epsilon$ with $0<\epsilon<1$, one has

$$
\begin{aligned}
\int_{\mathfrak{n}} \prod_{j=1}^{s}\left|g_{j}(\boldsymbol{\alpha}, P)\right| & \prod_{i=r+1}^{R}\left|K\left(\alpha_{i}\right)\right| d \boldsymbol{\alpha} \\
& \ll\left(\sup _{\alpha \in \mathfrak{n}} \prod_{j=1}^{s}\left|g_{j}(\boldsymbol{\alpha}, P)\right|\right)^{\epsilon} \int_{\mathfrak{d}} \prod_{j=1}^{s}\left|g_{j}(\boldsymbol{\alpha}, P)\right|^{1-\epsilon} \prod_{i=r+1}^{R}\left|K\left(\alpha_{i}\right)\right| d \boldsymbol{\alpha} .
\end{aligned}
$$

It follows from trivial estimates that one has

$$
\begin{aligned}
\int_{\mathfrak{n}} \prod_{j=1}^{s}\left|g_{j}(\boldsymbol{\alpha}, P)\right| & \prod_{i=r+1}^{R}\left|K\left(\alpha_{i}\right)\right| d \boldsymbol{\alpha} \\
& \left.\ll\left(h(\mathfrak{n}, P) P^{s}\right)^{\epsilon}\left(P^{s-\ell R}\right)^{1-\epsilon} \int_{\mathfrak{d}}^{\ell R}\left|\prod_{j=1}\right| g_{j}(\boldsymbol{\alpha}, P)\right|^{1-\epsilon} \prod_{i=r+1}^{R}\left|K\left(\alpha_{i}\right)\right| d \boldsymbol{\alpha} .
\end{aligned}
$$

We may certainly choose a positive real number $\epsilon$ so that we have

$$
\ell(1-\epsilon)>4 \quad \text { if } \quad k=2 .
$$

Defining $C^{\prime}$ as in Lemma 6.1 and choosing $\tilde{C}_{2}$ to be sufficiently large, we may ensure that we have

$$
\ell \geq \min \left(2^{k}+1, k(\log k+\log \log k+2)+\frac{C^{\prime} k \log \log k}{\log k}+1\right) \quad \text { if } \quad k \geq 3 .
$$

Thus we may choose a positive real number $\epsilon$, small enough (in terms only of $k$ and $C)$ so that we have

$$
\ell(1-\epsilon) \geq \min \left(2^{k}, k(\log k+\log \log k+2)+\frac{C^{\prime} k \log \log k}{\log k}\right) \quad \text { if } \quad k \geq 3 .
$$

In each of the cases, we denote our particular choice of $\epsilon$ by $\nu$. Now one can join the proof of Lemma 8 of [10] after equation (66), and then follow the remainder of that proof with only slight adjustments. The bounds (6.4) and (6.5) are the crucial bounds that we need to apply Lemma 6.1 We omit the details.

Now we can wrap up our work on the minor arcs. We have the following lemma. 
Lemma 6.3. Suppose that we are in the setting of Theorem 1.2 and that the coefficient matrix $A$ of the system $\mathbf{F}$ satisfies the conditions (3.2) and (3.3). Choose a function $T(P)$ as in Lemma (5.5). Define the exponential sums $g_{j}(\boldsymbol{\alpha})$ as in (4.8), with $\eta$ sufficiently small, the region $\mathfrak{m}$ as in (4.13), and the function $K$ as in (4.2). Then one has

$$
\int_{\mathfrak{m}} \prod_{j=1}^{s}\left|g_{j}(\boldsymbol{\alpha})\right| \prod_{i=r+1}^{R}\left|K\left(\alpha_{i}\right)\right| d \boldsymbol{\alpha}=o\left(P^{s-R k}\right) .
$$

Proof. We simply apply Lemma 6.2 with the choices $\mathfrak{n}=\mathfrak{m}$ and $d(P)=0$. We have $h(\mathfrak{m}, P)=o(1)$ by Lemma 5.5. Thus the proof of Lemma 6.3 is complete.

\section{The Trivial Arcs}

In this section we show that the contribution from the trivial arcs to the integral in (4.9) is $o\left(P^{s-R k}\right)$, which is now easy to do, having done the necessary work above. We have the following lemma.

Lemma 7.1. Suppose that we are in the setting of Theorem 1.2 and that the coefficient matrix $A$ of the system $\mathbf{F}$ satisfies (3.2) and (3.3). Choose a function $T(P)$ as in Lemma (5.5). Define the exponential sums $g_{j}(\boldsymbol{\alpha})$ as in (4.8) with $\eta$ sufficiently small, the region $\mathfrak{t}$ as in (4.14), and the function $K$ as in (4.2). Then one has

$$
\int_{\mathfrak{t}} \prod_{j=1}^{s}\left|g_{j}(\boldsymbol{\alpha})\right| \prod_{i=r+1}^{R}\left|K\left(\alpha_{i}\right)\right| d \boldsymbol{\alpha}=o\left(P^{s-R k}\right) .
$$

Proof. We apply Lemma 6.2 with the choices $\mathfrak{n}=\mathfrak{t}$ and $d(P)=T(P)$. We have $h(\mathfrak{m}, P)=O(1)$ by trivial estimates. Thus we obtain

$$
\int_{\mathfrak{t}} \prod_{j=1}^{s}\left|g_{j}(\boldsymbol{\alpha})\right| \prod_{i=r+1}^{R}\left|K\left(\alpha_{i}\right)\right| d \boldsymbol{\alpha} \ll(T(P))^{-1} P^{s-R k},
$$

which by (5.15) of Lemma 5.5 is $o\left(P^{s-R k}\right)$. Thus the proof of Lemma 7.1 is complete.

\section{The Major Arcs}

We now treat the major arcs. Our goal is to show that for large $P$ we have

$$
\int_{\mathcal{M}} \prod_{j=1}^{s} g_{j}(\boldsymbol{\alpha}) \prod_{i=r+1}^{R} K\left(\alpha_{i}\right) d \boldsymbol{\alpha} \gg_{\mathbf{F}} P^{s-R k} .
$$

8.1. Approximation on the major arcs. We start our treatment of the major arcs by approximating the functions $g_{j}(\boldsymbol{\alpha})$ by auxiliary functions. We need some notation before we do so. We define Dickman's function $\rho$ by the conditions

$$
\begin{array}{ll}
\rho(u)=0 & \text { for } u \leq 0, \\
\rho(u)=1 & \text { for } 0<u \leq 1, \\
u \rho^{\prime}(u)=-\rho(u-1) & \text { for } u>1, \\
\rho \text { is continuous } & \text { for } u>0, \\
\rho \text { is differentiable } & \text { for } u>1 .
\end{array}
$$


Also, for real numbers $\beta$, define the function

$$
\omega(\beta)=\frac{1}{k} \int_{0}^{P^{k}} x^{(1 / k)-1} \rho\left(\frac{\log x}{k \log P}\right) e(\beta x) d x .
$$

For real vectors $\boldsymbol{\beta} \in \mathbb{R}^{R}$, define $\Lambda_{j}(\boldsymbol{\beta})$ as in (4.7), and write

$$
\omega_{j}(\boldsymbol{\beta})=\omega\left(\Lambda_{j}(\boldsymbol{\beta})\right) \quad \text { for } \quad 1 \leq j \leq s .
$$

Also, for integers $q$ and $a$ with $q \geq 1$, define

$$
S(q, a)=\sum_{x=1}^{q} e\left(\frac{a x^{k}}{q}\right) .
$$

We now collect some results, given by Brüdern and Cook [5], in the following lemma.

Lemma 8.1. Define $g(\alpha)$ as in (4.6) and $\omega(\beta)$ as in (8.2). Suppose that a and $q$ are integers with $q \geq 1$, and that $\beta$ is a real number. Then one has

$$
g\left(\frac{a}{q}+\beta\right)=q^{-1} S(q, a) \omega(\beta)+O\left(\frac{P}{\log P}\left(q+P^{k}|\beta|\right)\right)
$$

and

$$
\omega(\beta) \ll \min \left(P,|\beta|^{-1 / k}\right) .
$$

Proof. The first result is simply equation (29) of [5]. The second result is essentially the third centered equation on page 135 of 5 .

We note that, as remarked by Brüdern and Cook, $a$ and $q$ are not required to be relatively prime. We now state the central lemma of the section, which is very similar to Lemma 4.4 of [16].

Lemma 8.2. Suppose that we are in the setting of Theorem 1.2 and that the coefficient matrix $A$ of the system $\mathbf{F}$ satisfies (3.2) and (3.3), and that there is a real vector $\mathbf{z}$ satisfying (3.6). Define the so-called singular series $\mathfrak{S}$ by

$$
\begin{aligned}
& \mathfrak{S}=1 \quad \text { if } r=0 \text {, and } \\
& \mathfrak{S}=\sum_{q=1}^{\infty} \sum_{\substack{\mathbf{a}(\bmod q) \\
\left(a_{1}, \ldots, a_{r}, q\right)=1}} q^{-s} \prod_{j=1}^{s} S\left(q, \Lambda_{j}^{(r)}(\mathbf{a})\right) \quad \text { for } \quad 1 \leq r \leq R,
\end{aligned}
$$

and the singular integral $\mathcal{I}(P)$ by

$$
\mathcal{I}(P)=\int_{\mathbb{R}^{R}}\left(\prod_{j=1}^{s} \omega_{j}(\boldsymbol{\beta})\right)\left(\prod_{i=r+1}^{R} K\left(\beta_{i}\right)\right) d \boldsymbol{\beta} .
$$

Fix any positive real number $\epsilon$. Then if $P$ is a sufficiently large positive real number, we have

$$
\begin{aligned}
\int_{\mathcal{M}}\left(\prod_{j=1}^{s} g_{j}(\boldsymbol{\alpha})\right)\left(\prod_{i=r+1}^{R} K\left(\alpha_{i}\right)\right) d \boldsymbol{\alpha}-\mathfrak{S I}(P) \\
\ll P^{s-R k}\left((\log P)^{2 B(R+1)-1}+(\log P)^{B(2-(\ell / k)+\epsilon)}\right) .
\end{aligned}
$$


Proof. There are three steps of the proof. One first approximates each function $g_{j}(\boldsymbol{\alpha})$ by terms of the form $q^{-1} S\left(q, \Lambda_{j}^{(r)}(\mathbf{a})\right) \omega_{j}(\boldsymbol{\beta})$ on each of the major arcs, then one extends the integration over each major arc to all of $\mathbb{R}^{R}$, and then one extends the sum over $q$ to all positive integers $q$. The argument closely follows the proof of Lemma 4.4 of [16]. One major difference involves the use of the approximations given in Lemma 8.1. Since we are using exponential sums over smooth numbers, we need to use these approximations instead of more standard results for exponential sums over complete intervals. Finally, we observe that the only condition we really need on $\ell$ for the purposes of this lemma is the bound $\ell \geq 2 k+1$.

Now we turn to consideration of the singular series $\mathfrak{S}$ and the singular integral $\mathcal{I}(P)$. In particular, we shall show that we have $\mathfrak{S} \gg 1$ and also that we have $\mathcal{I}(P) \gg P^{s-R k}$ for sufficiently large $P$. We first treat the singular series.

8.2. The singular series. We give some definitions. Suppose that $G_{1}, G_{2}, \ldots, G_{r}$ are $r$ integral diagonal forms in $s$ variables with coefficient matrix $B$, with entries $d_{i j}$. In Section 8.2 we consider integral forms, and of course we assume that $r \geq 1$ holds throughout this section. by

We then define the singular series $\mathfrak{S}(\mathbf{G})$ associated with this system of $r$ forms

$$
\mathfrak{S}=\mathfrak{S}(\mathbf{G})=\sum_{q=1}^{\infty} \sum_{\substack{\mathbf{a}(\bmod q) \\\left(a_{1}, \ldots, a_{r}, q\right)=1}} q^{-s} \prod_{j=1}^{s} S\left(q, M_{j}(\mathbf{a})\right)
$$

where we set

$$
M_{j}(\mathbf{a})=\sum_{i=1}^{r} d_{i j} a_{i} \quad \text { for } \quad 1 \leq j \leq s .
$$

We return to the notation of the rest of the paper for a moment. Observe that the definition of $\mathfrak{S}$ given here coincides with the definition (8.5) in the case $r=R$. Moreover, in general, when $1 \leq r \leq R$ holds, the first singular series is exactly the latter singular series, where the latter is associated with the first $r$ forms $F_{1}, \ldots, F_{r}$. Note also that the first singular series is independent of the $R-r$ forms $F_{r+1}, \ldots, F_{R}$.

Suppose that $p$ is a prime and $n$ is a positive integer. Then we say that an integral vector $\mathbf{x}=\left(x_{1}, x_{2}, \ldots, x_{s}\right)$ is a solution of rank $r(\bmod p)$ of the system of congruences

$$
G_{1}(\mathbf{x}) \equiv G_{2}(\mathbf{x}) \equiv \cdots \equiv G_{r}(\mathbf{x}) \equiv 0 \quad\left(\bmod p^{n}\right)
$$

if there is a subset $J \subseteq\{1,2, \ldots, s\}$ with $|J|=r$ such that one has $p \nmid \operatorname{det}\left(B_{J}\right)$ and $p \nmid \prod_{j \in J} x_{j}$. Also, for any prime $p$ and any positive integer $n$, we define $M\left(p^{n}, \mathbf{G}\right)$ to be the number of solutions $\mathbf{x}\left(\bmod p^{n}\right)$ of the system (8.8).

Now we shall define the concept of a normalized system of forms. We follow Low, Pitman and Wolff [13] closely, but we need a slightly more general notion. We essentially want to define a notion of a system such that a related system, which results after setting all but some subset of $t r$ of the variables equal to zero, is normalized in the original sense of Low, Pitman and Wolff. 
Suppose that the coefficient matrix $B$ contains $t$ disjoint nonsingular $r \times r$ submatrices $B_{1}, B_{2}, \ldots, B_{t}$. To be clear, by this we mean that there is some permutation of the columns of $B$ so that the first $r$ columns form a nonsingular submatrix, the second $r$ columns form a nonsingular submatrix, and so on, through the $t^{\text {th }}$ set of $r$ columns. We define

$$
\Delta=\Delta(\mathbf{G})=\prod_{v=1}^{t}\left|\operatorname{det}\left(B_{v}\right)\right| .
$$

Now let $\mathbf{j}=\left(j_{1}, j_{2}, \ldots, j_{t r}\right)$ be the ordered $(t r)$-tuple such that the particular matrix $\left[B_{1} B_{2} \ldots B_{t}\right]$ is the submatrix of $B$ consisting of the columns of $B$ indexed in order by $j_{1}, j_{2}, \ldots, j_{t r}$; that is, we define $\mathbf{j}$ so that for $1 \leq v \leq t$ and $1 \leq h \leq r$, the $j_{v(r-1)+h}^{\text {th }}$ column of $B$ is the $h^{\text {th }}$ column of $B_{v}$. Observe that the definition of $\Delta$ depends on $\mathbf{j}$. Also set

$$
J=\left\{j_{1}, j_{2}, \ldots, j_{t r}\right\} .
$$

Suppose that $p$ is a prime dividing $\Delta$. Here we define, following [13] closely, a $p$-operation on the forms $G_{1}, \ldots, G_{r}$ as a transformation that produces integral forms $H_{1}, \ldots, H_{r}$, and has the following steps:

(i) Pre-multiply $B$ by an integral unimodular matrix $U$ with entries in the set $\{0,1, \ldots, p-1\}$;

(ii) Next, multiply at most $t r-r$ of the columns of $U B_{J}$ by $p^{k}$ and multiply any of the columns of $U B_{\{1, \ldots, s\} \backslash J}$ by $p^{k}$;

(iii) Then divide $g$ of the rows by $p$, where we have $1 \leq g \leq r$.

As discussed in [13], step (i) corresponds to adding linear combinations of some of the forms to one or more of the other forms. Step (ii), on the other hand, corresponds to writing $x_{j}=p y_{j}$ in each column $j$ that one multiplies by $p^{k}$, and then trying to solve the new inequalities in the variables $y_{j}$. Step (iii) corresponds to dividing $g$ of the $r$ equations by $p$. One can check, as in [13], that a $p$-operation is possible for all primes $p$ that divide $\Delta$.

Note that for the resulting system $\mathbf{H}$, we have

$$
\Delta(\mathbf{H})=p^{m} \Delta(\mathbf{G})
$$

for some integer $m$. We say that such a $p$-operation is permissible if one has $m<0$.

Observe that, upon performing permissible $p$-operations for any of the primes $p$ dividing $\Delta(\mathbf{G})$, we can find a system $\mathbf{H}$ that can be obtained from the original system $\mathbf{G}$ via a finite sequence of permissible $p$-operations and such that $\Delta(\mathbf{H})$ is minimal. If $\mathbf{G}$ is a system of $r$ integral forms as above, such that $\Delta(\mathbf{G})$ cannot be reduced by any permissible $p$-operations, then we say that $\mathbf{G}$ is a $(\mathbf{j}, t)$-normalized system. Finally, if $\mathbf{G}$ is a system of $r$ integral forms in exactly $t r$ variables, then we simply say that $\mathbf{G}$ is a normalized system. We note that, in this case, our definition clearly agrees with the definition given for a normalized system in [13].

We make one other observation. Suppose that, as above, the coefficient matrix $B$ of a system $\mathbf{G}$, in $s$ variables with coefficients $d_{i j}$, contains $t$ disjoint nonsingular $r \times r$ submatrices $B_{1}, B_{2}, \ldots, B_{t}$, and define $\mathbf{j}$ and $J$ as above. Then, we define the system $\mathbf{G}^{*}$ in $t r$ variables, by defining, for $\mathbf{y} \in \mathbb{Z}^{t r}$ and for $1 \leq i \leq r$, the forms

$$
G_{i}^{*}(\mathbf{y})=\sum_{n=1}^{t r} d_{i j_{n}} y_{j_{n}}^{k} .
$$


Note that this is simply the system obtained by setting all variables with indices $j \notin J$ equal to 0 , and subsequently reordering the variables. Observe that the coefficient matrix $B^{*}$, say, of the system $\mathbf{G}^{*}$ has the form

$$
B^{*}=\left[\begin{array}{llll}
B_{1} & B_{2} & \ldots & B_{t}
\end{array}\right] .
$$

Suppose now that a system $\mathbf{H}$ can be obtained from the system $\mathbf{G}$ after a finite sequence of permissible $p$-operations, and suppose that $\mathbf{H}$ is $(\mathbf{j}, t)$-normalized. Then consider the system $\mathbf{H}^{*}$, defined as in (8.9). We can see that the same $p$-operations (restricted to the columns $j \in J$ ) allow one to obtain $\mathbf{H}^{*}$ from the system $\mathbf{G}^{*}$; after all, the variables of $\mathbf{G}^{*}$ are a subset of those that appear in $\mathbf{G}$. Each operation is certainly still permissible, since the definition of $\Delta$ involves only the columns $j \in J$. If one could reduce $\Delta\left(\mathbf{H}^{*}\right)$ via a permissible $p$-operation, then we could simply extend step (ii) and multiply all of the columns of $U B_{\{1, \ldots, s\} \backslash J}$ by $p^{k}$. This would give a permissible $p$-operation for the system $\mathbf{H}$, which contradicts our assumption that $\mathbf{H}$ is $(\mathbf{j}, t)$-normalized. So if $\mathbf{H}$ is $(\mathbf{j}, t)$-normalized, then it follows that $\mathbf{H}^{*}$ is normalized, and moreover, if $\mathbf{H}$ results from $\mathbf{G}$ after a finite sequence of permissible $p$-operations, then $\mathbf{H}^{*}$ results from $\mathbf{G}^{*}$ from the same sequence of $p$-operations.

We can now state the following lemma, which is a step towards bounding the singular series below. We do slightly more than we need to, in the hope that it will be useful for future workers. For this reason, we state the lemma in a self-contained manner.

Lemma 8.3. Suppose that $r, k$ and $s$ are positive integers with $k \geq 2$, and suppose for $1 \leq i \leq r$ that

$$
D_{i}(\mathbf{x})=d_{i 1} x_{1}^{k}+d_{i 2} x_{2}^{k}+\ldots+d_{i s} x_{s}^{k}
$$

is an integral diagonal form of degree $k$. Suppose that $s$ satisfies $s \geq t r$, where $t$ is an integer satisfying

$$
t \geq 2 k+1
$$

Suppose that the coefficient matrix $C$ of the system $\mathbf{D}$ contains $t$ disjoint nonsingular $r \times r$ submatrices $C_{1}, C_{2}, \ldots, C_{t}$, and define

$$
\Delta(\mathbf{D})=\prod_{v=1}^{t}\left|\operatorname{det}\left(C_{v}\right)\right| .
$$

Also, define the singular series $\mathbf{S}(\mathbf{D})$ as in (8.7). Suppose that the following property, which we denote by $P(t, k, r)$, holds:

Given any system of $r$ integral diagonal forms $G_{1}, G_{2}, \ldots, G_{r}$ of degree $k$ in tr variables, with coefficient matrix $B$ which consists of $t$ disjoint nonsingular $r \times r$ submatrices and such that the system $\mathbf{G}$ is normalized, then for every prime $p$ and every positive integer $n$, there is a solution $\mathbf{x}$ of rank $r(\bmod p)$ of the system of congruences

$$
G_{1}(\mathbf{x}) \equiv G_{2}(\mathbf{x}) \equiv \cdots \equiv G_{r}(\mathbf{x}) \equiv 0 \quad\left(\bmod p^{n}\right) .
$$

Then the series $\mathbf{S}(\mathbf{D})$ converges absolutely and one has

$$
\mathfrak{S}(\mathbf{D}) \gg_{\mathbf{D}} 1 .
$$

If one also has

$$
t \geq k r+k+1
$$


then there exists a constant $c(k, r, s)$ that depends only on $k, r$, and $s$, such that one has

$$
\mathfrak{S}(\mathbf{D}) \geq c(k, r, s)(\Delta(\mathbf{D}))^{-3 s} .
$$

Proof. We first give some more notation. For any prime $p$, define $\gamma=\gamma(k, p)$ by choosing $\tau$ to satisfy $p^{\tau} \| k$, and setting

$$
\gamma=\left\{\begin{array}{cll}
1 & \text { if } & \tau=0 \\
\tau+1 & \text { if } & \tau>0 \text { and } p>2 \\
\tau+2 & \text { if } & \tau>0 \text { and } p=2
\end{array}\right.
$$

Also, for any prime $p$, we define

$$
\chi_{\mathbf{D}}(p)=\lim _{n \rightarrow \infty} \frac{M\left(p^{n}, \mathbf{D}\right)}{p^{n(s-r)}} .
$$

As in Chapter 5 of [ [6], and using also Lemma 2.10 of [15], one may see that this limit exists, that $\mathfrak{S}(\mathbf{D})$ converges absolutely, and that $\mathfrak{S}(\mathbf{D})$ is equal to an absolutely convergent product, that is, we have

$$
\mathfrak{S}(\mathbf{D})=\prod_{p} \chi_{\mathbf{D}}(p) .
$$

(We note that it is in this argument that one uses the condition $t \geq 2 k+1$, and that the rate at which the product converges depends on $\mathbf{D}$.)

Now define $\mathbf{D}^{*}$ as in (8.9), and define $\mathbf{j}$ and $J$ as in the discussion above. We may find a $(\mathbf{j}, t)$-normalized system $\mathbf{G}$, which can be obtained from $\mathbf{D}$ after a finite sequence of permissible $p$-operations. As we have noted above, $\mathbf{G}^{*}$ is then a normalized system, which one obtains from $\mathbf{D}^{*}$ after (essentially) the same permissible p-operations.

Since property $P(t, k, r)$ holds, there is, for all $p$ and $n$, a solution $\mathbf{w}$ of rank $r$ $(\bmod p)$ of the system of congruences $\mathbf{G}^{*}(\mathbf{w}) \equiv \mathbf{0}\left(\bmod p^{n}\right)$. By the way $\mathbf{G}^{*}$ was defined, one can see that if $\mathbf{y}=\left(y_{1}, y_{2}, \ldots, y_{s}\right)$ is defined by

$$
y_{j}=\left\{\begin{array}{ccc}
w_{j} & \text { if } & j \in J \\
0 & \text { if } & j \notin J
\end{array}\right.
$$

then we have that $\mathbf{y}$ is a solution of rank $r(\bmod p)$ of the system of congruences $G(\mathbf{y}) \equiv 0\left(\bmod p^{n}\right)$. In particular, this holds for $n=\gamma$. We may thus apply Lemma 6 of [13] to the system $\mathbf{G}$, whence we have

$$
M\left(p^{n}, \mathbf{G}\right) \geq p^{(s-r)(n-\gamma)} \quad \text { for } n>\gamma .
$$

From this fact we will deduce a lower bound for $M\left(p^{n}, \mathbf{D}\right)$.

To this end, suppose for some positive integer $n$ that $\mathbf{y} \in \mathbb{Z}^{s}$ is a solution of the congruences

$$
G_{1}(\mathbf{y}) \equiv G_{2}(\mathbf{y}) \equiv \cdots \equiv G_{r}(\mathbf{y}) \equiv 0 \quad\left(\bmod p^{n}\right) .
$$

Recall that the system $\mathbf{G}$ resulted from $\mathbf{D}$ after a finite sequence of permissible $q$ operations. Let $\mathbf{H}$ be a system such that $\mathbf{G}$ arises from $\mathbf{H}$ after a single permissible $q$-operation. Let $I$ be the subset of $\{1,2, \ldots, s\}$ consisting of the columns affected by step (ii) of this $q$-operation, that is, let $I$ consist of the indices such that the 
corresponding columns in step (ii) are multiplied by $q^{k}$. Then define the vector $\mathbf{x}=\left(x_{1}, x_{2}, \ldots, x_{s}\right)$ by setting

$$
x_{j}=\left\{\begin{array}{ccc}
q y_{j} & \text { if } & j \in I \\
y_{j} & \text { if } & j \notin I .
\end{array}\right.
$$

We show that one has

$$
H_{1}(\mathbf{x}) \equiv H_{2}(\mathbf{x}) \equiv \cdots \equiv H_{r}(\mathbf{x}) \equiv 0 \quad\left(\bmod p^{n}\right) .
$$

To see this, let $\mathbf{H}^{(i)}$ and $\mathbf{H}^{(i i)}$ be the systems that result after steps (i) and (ii) of the $q$-operation, respectively. We certainly have $\mathbf{H}^{(i i)}(\mathbf{y}) \equiv \mathbf{0}\left(\bmod p^{n}\right)$; indeed, some of these forms are congruent to $0\left(\bmod p^{n} q\right)$. Then observe that we have $\mathbf{H}^{(i i)}(\mathbf{y})=\mathbf{H}^{(i)}(\mathbf{x})$, whence $\mathbf{H}^{(i)}(\mathbf{x}) \equiv \mathbf{0}\left(\bmod p^{n}\right)$ holds. Since the matrix $U$ is unimodular, so that in particular its determinant is not divisible by $p$, one has that (8.13) holds. Thus any solution $\mathbf{y}$ of $\mathbf{G}(\mathbf{y}) \equiv \mathbf{0}\left(\bmod p^{n}\right)$ gives rise to a solution $\mathbf{x}$ of $\mathbf{H}(\mathbf{x}) \equiv \mathbf{0}\left(\bmod p^{n}\right)$. If $q \neq p$ holds, we therefore have

$$
M\left(p^{n}, \mathbf{H}\right) \geq M\left(p^{n}, \mathbf{G}\right) .
$$

If $q=p$ holds, we might have some reduction in the number of solutions, because multiplication by $p$ in $\mathbb{Z} / p^{n} \mathbb{Z}$ has kernel of size $p$, but we certainly have

$$
M\left(p^{n}, \mathbf{H}\right) \geq \frac{M\left(p^{n}, \mathbf{G}\right)}{p^{s-r}} .
$$

So, by repeating this analysis for each permissible $q$-operation, one can see that if $\mathbf{G}$ is a $(\mathbf{j}, t)$-normalized system arising from the system $\mathbf{F}$ after a finite sequence of permissible $q$-operations, then one has

$$
M\left(p^{n}, \mathbf{D}\right) \geq \frac{M\left(p^{n}, \mathbf{G}\right)}{p^{m(s-r)}} \quad \text { if } \quad p^{m} \| \Delta(\mathbf{D}) .
$$

Now the limit $\chi_{\mathbf{G}}(p)$ exists; this follows in much the same way as the corresponding fact for $\mathbf{D}$. It follows that we have

$$
\chi_{\mathbf{D}}(p) \geq \frac{\chi_{\mathbf{G}}(p)}{p^{m(s-r)}} \quad \text { if } \quad p^{m} \| \Delta(\mathbf{D}) .
$$

It follows from (8.12) and the definition of $\chi_{\mathbf{G}}(p)$ that for all primes $p$ we have

$$
\chi_{\mathbf{D}}(p) \geq p^{-\left(\gamma+\operatorname{ord}_{p}(\Delta(\mathbf{D}))\right)(s-r)} .
$$

Since the product $\prod_{p} \chi_{\mathbf{D}}(p)$ is absolutely convergent, there is a constant $c(\mathbf{D})$, which may depend on $\mathbf{D}$, such that we have

$$
\prod_{p>c(\mathbf{D})} \chi_{\mathbf{D}}(p) \geq \frac{1}{2}
$$

Thus, using also (8.15), we have

$$
\begin{aligned}
\mathfrak{S}(\mathbf{D}) & \geq \frac{1}{2} \prod_{p \leq c(\mathbf{D})} \chi_{\mathbf{D}}(p) \geq \frac{1}{2} \prod_{p \leq c(\mathbf{D})} p^{-\left(\gamma+\operatorname{ord}_{p}(\Delta(\mathbf{D}))\right)(s-r)} \\
& \geq \frac{1}{2}(\Delta(\mathbf{D}))^{r-s} \prod_{p \leq c(\mathbf{D})} p^{-\gamma(s-r)} \gg_{\mathbf{D}} 1 .
\end{aligned}
$$


Now suppose that we have $t \geq k r+k+1$. One can prove as in chapter 5 of $[\underline{6}$ that for primes $p$, one has

$$
\chi_{\mathbf{D}}(p)=1+\sum_{n=1}^{\infty} S\left(p^{n}\right)
$$

where for positive integers $n$, we define

$$
S\left(p^{n}\right)=p^{-n s} \sum_{\substack{\mathbf{a}\left(\bmod p^{n}\right) \\\left(a_{1}, \ldots, a_{r}, p\right)=1}} \prod_{j=1}^{s} S\left(p^{n}, M_{j}(\mathbf{a})\right) .
$$

Now suppose that $p \nmid \Delta(\mathbf{D})$. Suppose for $1 \leq v \leq t$ that $C_{v}$ consists of the columns $j_{v_{1}}, j_{v_{2}}, \ldots, j_{v_{r}}$, in that order. Then for a satisfying $\left(a_{1}, \ldots, a_{r}, p\right)=1$, there must exist some $j \in\left\{j_{v_{1}}, \ldots, j_{v_{r}}\right\}$ such that $p \nmid M_{j}(\mathbf{a})$, since we have $p \nmid$ $\operatorname{det}\left(C_{v}\right)$. Thus for any prime $p$ with $p \nmid \Delta(\mathbf{D})$, and any positive integer $n$, by the standard estimate $S\left(p^{n}, a\right) \ll p^{n(1-(1 / k))}$, which holds for $(a, p)=1$, we have

$$
S\left(p^{n}\right) \ll p^{-n s} \sum_{\substack{\mathbf{a}\left(\bmod p^{n}\right) \\\left(a_{1}, \ldots, a_{r}, p\right)=1}} p^{n s-(n t / k)} \ll p^{n(r-(t / k))} .
$$

Combining this last bound with (8.16) and using $t \geq k r+k+1$ yields

$$
\chi_{\mathbf{D}}(p)-1 \ll \sum_{n=1}^{\infty} p^{n(r-(t / k))} \ll p^{-1-(1 / k)} \quad \text { for } \quad p \nmid \Delta(\mathbf{D}) .
$$

So there is a constant $C$ that depends only on $k, r$ and $t$ such that one has

$$
\left|\chi_{\mathbf{D}}(p)-1\right| \leq C p^{-1-(1 / k)} \quad \text { for } \quad p \nmid \Delta(\mathbf{D}) .
$$

Now, because $\sum_{p} C p^{-1-(1 / k)}$ converges, there is a constant $\tilde{C}$ that depends only on $k, r$ and $t$ such that one has $1-C p^{-1-(1 / k)}>0$ for $p>\tilde{C}$ and

$$
\prod_{p>\tilde{C}}\left(1-C p^{-1-(1 / k)}\right) \geq \frac{1}{2} .
$$

Now for all $p$ we have $\chi_{\mathbf{D}}(p)>0$ from (8.15); so by 8.17) we have

$$
\begin{aligned}
\prod_{p} \chi_{\mathbf{D}}(p) & =\prod_{p \leq \tilde{C}} \chi_{\mathbf{D}}(p) \prod_{\substack{p>\tilde{C} \\
p \mid \Delta(\mathbf{D})}} \chi_{\mathbf{D}}(p) \prod_{\substack{p>\tilde{C} \\
p \nmid \Delta(\mathbf{D})}} \chi_{\mathbf{D}}(p) \\
& \geq \prod_{p \leq \tilde{C}} \chi_{\mathbf{D}}(p) \prod_{\substack{p>\tilde{C} \\
p \mid \Delta(\mathbf{D})}} \chi_{\mathbf{D}}(p) \prod_{\substack{p>\tilde{C} \\
p \nmid \Delta(\mathbf{D})}}\left(1-C p^{-1-(1 / k)}\right) \\
& \geq \frac{1}{2} \prod_{p \leq \tilde{C}} \chi_{\mathbf{D}}(p) \prod_{\substack{p>\tilde{C} \\
p \mid \Delta(\mathbf{D})}} \chi_{\mathbf{D}}(p) .
\end{aligned}
$$


It follows from (8.15) that we have

$$
\begin{aligned}
\prod_{p} \chi_{\mathbf{D}}(p) & \geq \quad \frac{1}{2} \prod_{\substack{p \leq \tilde{C} \\
p \nmid \Delta(\mathbf{D})}} p^{-\gamma(s-r)} \prod_{p \mid \Delta(\mathbf{D})} p^{-\left(\gamma+\operatorname{ord}_{p}(\Delta(\mathbf{D}))\right)(s-r)} \\
& \geq \\
& \frac{1}{2}\left(\prod_{p \leq \tilde{C}} p^{-\gamma(s-r)}\right)(\Delta(\mathbf{D}))^{r-s} \prod_{p \mid \Delta(\mathbf{D})} p^{-\gamma(s-r)} \\
& \gg_{k, r, s}(\Delta(\mathbf{D}))^{r-s}(\Delta(\mathbf{D}))^{2(r-s)}
\end{aligned}
$$

This completes the proof of Lemma 8.3 .

Now we give another lemma that builds on the above lemma and completes the treatment of the singular series for the cases $k \geq 3$. As in the case of Lemma 8.3, we do slightly more than what we will need, and we state the lemma in a self-contained fashion.

Lemma 8.4. Suppose that $r, k$, and $s$ are positive integers, and suppose for $1 \leq$ $i \leq r$ that

$$
F_{i}(\mathbf{x})=\lambda_{i 1} x_{1}^{k}+\lambda_{i 2} x_{2}^{k}+\ldots+\lambda_{i s} x_{s}^{k}
$$

is an integral diagonal form of degree $k$. Suppose that the coefficient matrix $A$ of the system $\mathbf{F}$ contains $\ell$ disjoint nonsingular $r \times r$ submatrices $A_{1}^{(r)}, A_{2}^{(r)}, \ldots, A_{\ell}^{(r)}$, where $\ell$ is a positive integer satisfying

$$
\ell \geq 2 k+1 \text {. }
$$

Define $\Delta=\prod_{v=1}^{\ell}\left|\operatorname{det}\left(A_{v}^{(r)}\right)\right|$. Define $\mathfrak{S}=\mathfrak{S}(\mathbf{F})$ as in (8.7). Finally, suppose that one of the two following statements holds.

(i) $k$ is odd, and $k \geq 3$ holds, and one has $\ell \geq k m_{0}$, where $m_{0}$ is the least positive integer $m$ such that one has

$$
2^{m-2} \geq \min \left\{m^{2}(2 k)^{r},\left(3 r k^{2}\right)^{r}\right\} .
$$

(ii) $k \geq 3$ holds, and one has

$$
\ell>k\left[48 k^{2} \log 3 r k^{2}\right] .
$$

Then one has

$$
\mathfrak{S}(\mathbf{F}) \gg_{\mathbf{F}} 1 \text {. }
$$

Moreover, if

$$
\ell \geq k r+k+1
$$

holds, then there exists a positive real constant $c(k, r, s)$ that depends only on $k, r$ and $s$ such that one has

$$
\mathfrak{S}(\mathbf{F}) \geq c(k, r, s) \Delta^{-3 s} .
$$

Moreover, we note that if $k$ is odd with $k \geq 3$, then there exists an absolute positive real constant $C$ such that condition (i) holds if one has

$$
\ell \geq \frac{r k \log 2 k}{\log 2}+C k \log (r \log 2 k) \text {. }
$$


Proof. The last statement of the lemma can be checked with a straightforward computation.

Thus to prove the lemma, we need only check that the condition $P(\ell, k, r)$ of Lemma 8.3 holds for our choices of $\ell$. So suppose that $\mathbf{G}$ is a normalized system in $\ell r$ variables. For the case in which condition (i) holds, we may apply Theorem 1(ii) of 13 to see that for any positive integer $n$, the system $\mathbf{G}(\mathbf{x}) \equiv \mathbf{0}\left(\bmod p^{n}\right)$ has a solution $\mathbf{x}$ of rank $r(\bmod p)$. On the other hand, for the case in which condition (ii) holds, we may apply Theorem 3(i) of [13].

Thus in either case, the condition $P(\ell, k, r)$ of Lemma 8.3 holds, whence Lemma 8.4 follows.

Now we give a lemma to treat the singular series in the case $k=2$. We observe that one could surely obtain a result that is better for large $r$, but we choose not to pursue this here.

Lemma 8.5. Suppose that $r$ and $s$ are positive integers, and suppose for $1 \leq i \leq r$ that

$$
F_{i}(\mathbf{x})=\lambda_{i 1} x_{1}^{2}+\lambda_{i 2} x_{2}^{2}+\ldots+\lambda_{i s} x_{s}^{2}
$$

is an integral diagonal quadratic form. Suppose that the coefficient matrix A, defined as in (1.3), contains $\ell$ disjoint nonsingular $r \times r$ submatrices, where $\ell$ is an integer satisfying

$$
\ell \geq \min \left(4 r^{2}+4 r+1,384 \log 16 r+5\right) .
$$

Define $\mathfrak{S}=\mathfrak{S}(\mathbf{F})$ as in (8.7). Then one has

$$
\mathfrak{S}(\mathbf{F}) \gg_{\mathbf{F}} 1 .
$$

Proof. If the first bound for $\ell$ holds, then it follows that any nontrivial complex linear combination of the forms $F_{1}, \ldots, F_{r}$ has rank at least $4 r^{2}+4 r+1$. By the theorem in [18], the singular series $\mathfrak{S}$ is positive and depends only on the forms $F_{1}, F_{2}, \ldots, F_{r}$. One can readily check that the singular series $\mathfrak{S}$ is defined in [18] in the same manner as we have defined it.

Suppose instead that the second bound for $\ell$ holds. We give a sketch of the proof in this case. We first seek an analogue of Lemma 12 of [13] for the case $k=2$, with $m \geq[192 \log 16 r+2]$. For primes $p$ with $p \leq 8 r^{2}$, say, it is easy to check that Lemma 5 of $[7$ provides an analogue of the desired type. To obtain an appropriate analogue of Lemma 12 of [13] for primes $p>8 r^{2}$, one applies an adaptation of Theorem 2 of 14, with, say, $c=4$; one can check that if one assumes that the matrix of coefficients contains $c+1$ nonsingular $r \times r$ submatrices, rather than assuming that the matrix is highly nonsingular, then the result still holds. (This can be seen by noting that the inequality $q_{i}(\mathbf{B})>c i$, which would still hold, is the key condition needed on page 339 of 14.)

In either case, we have an analogue of Lemma 12 of [13, and thus we can show that condition $P(\ell, k, r)$ holds, as in the proof of Theorem 1(ii) of [13].

Michael Knapp and Professor Wooley provided me with a proof of a result closely related to the second part of the above lemma, for which I am grateful. 
8.3. The singular integral. Recall that in (8.6) we defined the singular integral $\mathcal{I}(P)$ by

$$
\mathcal{I}(P)=\int_{\mathbb{R}^{R}}\left(\prod_{j=1}^{s} \omega_{j}(\boldsymbol{\beta})\right)\left(\prod_{i=r+1}^{R} K\left(\beta_{i}\right)\right) d \boldsymbol{\beta} .
$$

Our goal in this section is to demonstrate that for large positive $P$ we have the bound

$$
\mathcal{I}(P) \gg_{\mathbf{F}} P^{s-R k}
$$

Instead of using the traditional approach which uses Fourier's Integral Theorem, we use a method given by Schmidt [18. Below we follow parts of 18 very closely.

Much as in [18, for any positive real number $T$ and any real numbers $\alpha$ and $\beta$, we define

and

$$
K_{T}(\alpha)=K\left(\alpha T^{-1}\right)=\left(\frac{\sin \left(\pi \alpha T^{-1}\right)}{\pi \alpha T^{-1}}\right)^{2}
$$

From (4.3), one may readily deduce the following identity, which holds for all real numbers $\beta$, namely,

$$
\psi_{T}(\beta)=\int_{\mathbb{R}} e(\alpha \beta) K_{T}(\alpha) d \alpha
$$

We now define

$$
\mathcal{I}_{T}(P)=\int_{\mathbb{R}^{R}}\left(\prod_{j=1}^{s} \omega_{j}(\boldsymbol{\beta})\right)\left(\prod_{i=1}^{r} K_{T}\left(\beta_{i}\right)\right)\left(\prod_{i=r+1}^{R} K\left(\beta_{i}\right)\right) d \boldsymbol{\beta} .
$$

By (4.4) and a similar bound for $K_{T}(\alpha)$, the integral converges absolutely for each choice of $P$ and $T$.

We shall see that for fixed $P$, we have $\lim _{T \rightarrow \infty} \mathcal{I}_{T}(P)=\mathcal{I}(P)$, and we will also show that for large $T$, we have $\mathcal{I}_{T}(P) \gg_{\mathbf{F}} P^{s-R k}$. These two facts together establish the bound (8.18). To prove the first fact, we give a bound for the difference $\mathcal{I}_{T}(P)-$ $\mathcal{I}(P)$.

Lemma 8.6. Suppose that $T$ and $P$ are positive real numbers with $T \geq 1$ and $P \geq 1$. Suppose that $R, r, k$ and $s$ are integers with $R \geq 1,0 \leq r \leq R$ and $k \geq 2$. Suppose for $1 \leq i \leq R$ that

$$
F_{i}(\mathbf{x})=\lambda_{i 1} x_{1}^{2}+\lambda_{i 2} x_{2}^{2}+\ldots+\lambda_{i s} x_{s}^{2}
$$

is a real diagonal form of degree $k$ and that for $1 \leq i \leq r$, the form $F_{i}$ is integral. Assume that one has $\|\mathbf{F}\| \geq 1$. Suppose also that the coefficient matrix $A$ of the system $\mathbf{F}$ is as in (3.2) and satisfies (3.3), where one has

$$
\ell \geq k+1 \text {. }
$$

Define $\mathcal{I}(P)$ and $\mathcal{I}_{T}(P)$ as in (8.6) and (8.21), respectively. Then one has

$$
\mathcal{I}(P)-\mathcal{I}_{T}(P) \ll T^{-1 / k} P^{s-R k}\left(\|\mathbf{F}\|^{2 R} \prod_{v=1}^{\ell} \Delta_{v}^{-3 / \ell}+P^{k-\ell}\|\mathbf{F}\|^{(R \ell) / k} \prod_{v=1}^{\ell} \Delta_{v}^{-1 / k}\right) .
$$


We note that the implicit constant in Vinogradov's notation here depends at most on $R, r, k$ and $s$ and, in particular, does not depend on the coefficients of $\mathbf{F}$.

Proof. Observe first that, in the case $r=0$, we have $\mathcal{I}(P)=\mathcal{I}_{T}(P)$. So we can assume that we have $r \geq 1$. It follows from the definitions (8.6) and (8.21) that we have

$$
\mathcal{I}(P)-\mathcal{I}_{T}(P) \ll \int_{\mathbb{R}^{R}}\left(\prod_{j=1}^{s}\left|\omega_{j}(\boldsymbol{\beta})\right|\right)\left|1-\prod_{i=1}^{r} K_{T}\left(\beta_{i}\right)\right|\left(\prod_{i=r+1}^{R}\left|K\left(\beta_{i}\right)\right|\right) d \boldsymbol{\beta} .
$$

From the penultimate centered equation on page 305 of [18], one has

$$
1-\prod_{i=1}^{r} K_{T}\left(\beta_{i}\right) \ll T^{-2} \max _{1 \leq i \leq r}\left|\beta_{i}\right|^{2} \ll T^{-2}|\boldsymbol{\beta}|^{2} \quad \text { for } \quad|\boldsymbol{\beta}|<T,
$$

and for $|\boldsymbol{\beta}| \geq T$, one clearly has $\prod_{i=1}^{r} K_{T}\left(\beta_{i}\right) \ll 1$. Combining these bounds with the estimate for $\omega(\boldsymbol{\beta})$ given in Lemma 8.1, and the bound (4.4) for $K(\alpha)$, one has

$$
\begin{aligned}
\mathcal{I}(P)-\mathcal{I}_{T}(P) \ll T^{-2} P^{s-R \ell} \int_{|\boldsymbol{\beta}|<T} & \left(\prod_{j=1}^{R \ell} \min \left(P,\left|\Lambda_{j}(\boldsymbol{\beta})\right|^{-1 / k}\right)\right)|\boldsymbol{\beta}|^{2} d \boldsymbol{\beta} \\
& +P^{s-R \ell} \int_{|\boldsymbol{\beta}| \geq T} \prod_{j=1}^{R \ell} \min \left(P,\left|\Lambda_{j}(\boldsymbol{\beta})\right|^{-1 / k}\right) d \boldsymbol{\beta} .
\end{aligned}
$$

Consider the first integral on the right-hand side of 8.22). By Hölder's inequality, one has

$$
\begin{aligned}
\int_{|\boldsymbol{\beta}|<T}|\boldsymbol{\beta}|^{2} & \prod_{j=1}^{R \ell} \min \left(P,\left|\Lambda_{j}(\boldsymbol{\beta})\right|^{-1 / k}\right) d \boldsymbol{\beta} \\
\ll & \prod_{v=1}^{\ell}\left(\int_{|\boldsymbol{\beta}|<T}|\boldsymbol{\beta}|^{2} \prod_{j=(v-1) R+1}^{v R} \min \left(P,\left|\Lambda_{j}(\boldsymbol{\beta})\right|^{-1 / k}\right)^{\ell} d \boldsymbol{\beta}\right)^{1 / \ell} .
\end{aligned}
$$

For a fixed choice of $v$ with $1 \leq v \leq \ell$, one makes the change of variable $\gamma=$ $\left(\gamma_{1}, \ldots, \gamma_{R}\right)=U_{v}(\boldsymbol{\beta})$ given by $\gamma_{j}=\Lambda_{(v-1) R+j}(\boldsymbol{\beta})$ for $1 \leq j \leq R$, and obtains

$$
\begin{aligned}
\int_{|\boldsymbol{\beta}|<T}|\boldsymbol{\beta}|^{2} & \prod_{j=(v-1) R+1}^{v R} \min \left(P,\left|\Lambda_{j}(\boldsymbol{\beta})\right|^{-1 / k}\right)^{\ell} d \boldsymbol{\beta} \\
& \ll \Delta_{v}^{-1} \int_{\boldsymbol{\gamma} \in U_{v}([-T, T] R)}\left|U_{v}^{-1}(\boldsymbol{\gamma})\right|^{2} \prod_{j=1}^{R} \min \left(P,\left|\gamma_{j}\right|^{-1 / k}\right)^{\ell} d \boldsymbol{\gamma} .
\end{aligned}
$$

If $\boldsymbol{\gamma}=U_{v}(\boldsymbol{\beta})$, then one has

$$
|\gamma|=\left|U_{v}(\boldsymbol{\beta})\right| \leq R\|\mathbf{F}\| \cdot|\boldsymbol{\beta}|
$$


and by Cramer's rule and Hadamard's inequality, one has

$$
|\boldsymbol{\beta}|=\left|U_{v}^{-1}(\gamma)\right| \leq \frac{R^{R / 2}\|\mathbf{F}\|^{R-1}}{\Delta_{v}}|\gamma| .
$$

It follows that we have

$$
\begin{aligned}
\int_{|\boldsymbol{\beta}|<T}|\boldsymbol{\beta}|^{2} & \prod_{j=(v-1) R+1}^{v R} \min \left(P,\left|\Lambda_{j}(\boldsymbol{\beta})\right|^{-1 / k}\right)^{\ell} d \boldsymbol{\beta} \\
& \ll_{R} \Delta_{v}^{-3}\|\mathbf{F}\|^{2 R-2} \int_{|\boldsymbol{\gamma}| \leq R\|\mathbf{F}\| T}|\gamma|^{2} \prod_{j=1}^{R} \min \left(P,\left|\gamma_{j}\right|^{-1 / k}\right)^{\ell} d \boldsymbol{\gamma} .
\end{aligned}
$$

But one has

$$
\begin{aligned}
\int_{|\gamma| \leq R\|\mathbf{F}\| T}|\gamma|^{2} \prod_{j=1}^{R} \min \left(P,\left|\gamma_{j}\right|^{-1 / k}\right)^{\ell} d \boldsymbol{\gamma} \\
\ll \sum_{n=1}^{\lceil R\|\mathbf{F}\| T\rceil} n^{2} \int_{n-1 \leq|\gamma|<n} \prod_{j=1}^{R} \min \left(P,\left|\gamma_{j}\right|^{-1 / k}\right)^{\ell} d \gamma \\
\ll \sum_{n=1}^{\lceil R\|\mathbf{F}\| T\rceil} n^{2}\left(\int_{0}^{n} \min \left(P, \gamma^{-1 / k}\right)^{\ell} d \gamma\right)^{R-1} \int_{n-1}^{n} \min \left(P, \gamma^{-1 / k}\right)^{\ell} d \gamma \\
\ll \sum_{n=1}^{\lceil R\|\mathbf{F}\| T\rceil} n^{2} P^{(\ell-k)(R-1)} \min \left(P^{\ell-k},(n-1)^{-\frac{\ell}{k}}\right),
\end{aligned}
$$

whence we have

$$
\begin{aligned}
\int_{|\gamma| \leq R\|\mathbf{F}\| T}|\gamma|^{2} \prod_{j=1}^{R} & \min \left(P,\left|\gamma_{j}\right|^{-1 / k}\right)^{\ell} d \boldsymbol{\gamma} \\
& \ll P^{(\ell-k)(R-1)}\left(P^{\ell-k}+\sum_{n=1}^{\lceil R\|\mathbf{F}\| T\rceil} n^{2-(\ell / k)}\right) \\
& \ll P^{R(\ell-k)}+P^{(\ell-k)(R-1)} \sum_{n=1}^{\lceil R\|\mathbf{F}\| T\rceil} n^{1-(1 / k)} \\
& \ll P^{R(\ell-k)}(\|\mathbf{F}\| T)^{2-(1 / k)}
\end{aligned}
$$

since we have $\ell \geq k+1$ and $\|\mathbf{F}\| T \geq 1$.

Thus, by (8.22), (8.23) and (8.25), we have

$$
\begin{aligned}
\mathcal{I}(P)-\mathcal{I}_{T}(P) \ll\|\mathbf{F}\|^{2 R} T^{-1 / k} P^{s-R k} \prod_{v=1}^{\ell} \Delta_{v}^{-3 / \ell} \\
\\
+P^{s-R \ell} \int_{|\boldsymbol{\beta}| \geq T} \prod_{j=1}^{R \ell} \min \left(P,\left|\Lambda_{j}(\boldsymbol{\beta})\right|^{-1 / k}\right) d \boldsymbol{\beta} .
\end{aligned}
$$


By Hölder's inequality and a change of variable as above, we have

$$
\begin{aligned}
\mathcal{I}(P)-\mathcal{I}_{T}(P) \ll & \|\mathbf{F}\|^{2 R} T^{-1 / k} P^{s-R k} \prod_{v=1}^{\ell} \Delta_{v}^{-3 / \ell} \\
& +P^{s-R \ell} \prod_{v=1}^{\ell}\left(\Delta_{v}^{-1} \int_{|\gamma| \geq c_{\mathbf{F}, v} T} \prod_{j=1}^{R} \min \left(P,\left|\gamma_{j}\right|^{-1 / k}\right)^{\ell} d \boldsymbol{\gamma}\right)^{1 / \ell},
\end{aligned}
$$

where $c_{\mathbf{F}, v}$ is a positive constant which by (8.24) we may define by

$$
c_{\mathbf{F}, v}=\frac{\Delta_{v}}{R^{R / 2}\|\mathbf{F}\|^{R-1}} .
$$

It follows that one has

$$
\begin{aligned}
\int_{|\gamma| \geq c_{\mathbf{F}, v} T} \prod_{j=1}^{R} & \min \left(P,\left|\gamma_{j}\right|^{-1 / k}\right)^{\ell} d \boldsymbol{\gamma} \\
& \ll\left(\int_{0}^{\infty} \min \left(P,|\gamma|^{-1 / k}\right)^{\ell} d \gamma\right)^{R-1} \int_{c_{\mathbf{F}, v} T}^{\infty} \min \left(P,|\gamma|^{-1 / k}\right)^{\ell} d \gamma \\
& \ll P^{(\ell-k)(R-1)}\left(c_{\mathbf{F}, v} T\right)^{1-(\ell / k)} \\
& \ll P^{(\ell-k) R-\ell+k} \Delta_{v}^{1-(\ell / k)}\|\mathbf{F}\|^{(R \ell) / k} T^{-1 / k},
\end{aligned}
$$

since we have $\ell \geq k+1$. Combining the last two bounds completes the proof of Lemma 8.6

Now we prove a lemma which states that for $T$ and $P$ sufficiently large, the quantity $\mathcal{I}_{T}(P)$ is bounded below.

Lemma 8.7. Suppose that we are in the setting of Theorem 1.2 and that the coefficient matrix $A$ of the system $\mathbf{F}$ satisfies (3.2) and (3.3), and that there is a real vector $\mathbf{z}$ satisfying (3.6). Suppose also that one has $\|\mathbf{F}\| \geq 1$. Define $\Delta_{1}$ as in (3.3). Suppose that $T$ and $P$ are real numbers satisfying $T \geq 1$ and

$$
P \geq\left(\frac{12}{\delta} s^{R} R^{2 R}\|\mathbf{F}\|^{2 R} \max \left(\Delta_{1}^{-2}, 1\right)\right)^{1 / k}
$$

where $\delta$ is as in (3.6). Define $\mathcal{I}_{T}(P)$ as in (8.21). Then there is a constant $c_{1}=$ $c_{1}(\mathbf{F}, k, R, r, s, \delta, \mathbf{z})$ that does not depend on $T$ or $P$ such that one has

$$
\mathcal{I}_{T}(P) \geq c_{1} P^{s-R k} \text {. }
$$

Proof. Recalling the definition (8.3) of $\omega_{j}(\boldsymbol{\beta})$, we can write the absolutely convergent integral $\mathcal{I}_{T}(P)$ in the form

$$
\begin{array}{r}
\frac{1}{k^{s}} \int_{\mathbb{R}^{R}} \int_{\left[0, P^{k}\right]^{s}}\left(x_{1} x_{2} \cdots x_{s}\right)^{1 / k-1}\left(\prod_{j=1}^{s} \rho\left(\frac{\log x_{j}}{k \log P}\right)\right) e\left(\sum_{j=1}^{s} \Lambda_{j}(\boldsymbol{\beta}) x_{j}\right) \\
\times \prod_{i=1}^{r} K_{T}\left(\beta_{i}\right) \prod_{i=r+1}^{R} K\left(\beta_{i}\right) d \mathbf{x} d \boldsymbol{\beta} .
\end{array}
$$


Using the identities (8.20) and (4.3), and recalling the definition (3.4) of $L_{i}(\mathbf{x})$, we can rewrite $\mathcal{I}_{T}(P)$ as

$$
\frac{1}{k^{s}} \int_{\left[0, P^{k}\right]^{s}}\left(x_{1} \cdots x_{s}\right)^{1 / k-1} \prod_{j=1}^{s} \rho\left(\frac{\log x_{j}}{k \log P}\right)\left(\prod_{i=1}^{r} \psi_{T}\left(L_{i}(\mathbf{x})\right)\right)\left(\prod_{i=r+1}^{R} \psi\left(L_{i}(\mathbf{x})\right)\right) d \mathbf{x}
$$

$\mathcal{I}_{T}(P)$ is certainly larger than the corresponding integral over the smaller region $\left[\frac{\delta P^{k}}{2}, P^{k}\right]^{s}$, noting from Lemma $12.1(\mathrm{i})$ of $[21]$, and $(\underline{8.1}$, that the function $\rho$ is always nonnegative. For $P$ satisfying (8.26), one certainly has $P>(\delta / 2)^{-1 / k}$, whence for $x_{j}$ satisfying $\frac{\delta P^{k}}{2} \leq x_{j} \leq P^{k}$, it follows from (8.1) that one has

$$
\rho\left(\frac{\log x_{j}}{k \log P}\right)=1
$$

So we have

$$
\mathcal{I}_{T}(P) \geq \frac{1}{k^{s}} \int_{\left[\frac{\delta P^{k}}{2}, P^{k}\right]^{s}}\left(x_{1} x_{2} \cdots x_{s}\right)^{1 / k-1}\left(\prod_{i=1}^{r} \psi_{T}\left(L_{i}(\mathbf{x})\right)\right)\left(\prod_{i=r+1}^{R} \psi\left(L_{i}(\mathbf{x})\right)\right) d \mathbf{x} .
$$

Now define $\mathcal{R}_{P, T}$ to be the region

$$
\begin{aligned}
&\left\{\mathbf{x} \in\left[\frac{\delta P^{k}}{2}, P^{k}\right]^{s}:\right.\left|L_{i}(\mathbf{x})\right|<\frac{\min \left(1, \Delta_{1}^{1 / R}\right)}{3 T} \text { for } 1 \leq i \leq r \text { and } \\
&\left.\left|L_{i}(\mathbf{x})\right|<\frac{1}{3} \min \left(1, \Delta_{1}^{1 / R}\right) \text { for } r+1 \leq i \leq R\right\} .
\end{aligned}
$$

It follows from the definitions 8.19) and 4.3) of $\psi_{T}(\alpha)$ and $\psi(\alpha)$, respectively, that we have

$$
\mathcal{I}_{T}(P) \gg P^{s-s k} T^{r} \mu_{s}\left(\mathcal{R}_{P, T}\right)
$$

where $\mu_{s}$ denotes $s$-dimensional Euclidean measure.

We now make the linear change of variable $\mathbf{w}=V(\mathbf{x})$ given by

$$
w_{j}=\left\{\begin{array}{ccl}
L_{j}(\mathbf{x}) & \text { for } \quad 1 \leq j \leq R \\
x_{j} & \text { for } \quad R+1 \leq j \leq s .
\end{array}\right.
$$

Since $\Delta_{1}$ is nonzero, we can see that we have

$$
\mu_{s}\left(\mathcal{R}_{P, T}\right) \gg_{\mathbf{F}} \mu_{s}\left(\mathcal{S}_{P, T}\right)
$$

where we define $\mathcal{S}_{P, T}$ to be the region $V\left(\mathcal{R}_{P, T}\right)$. Note that $\mathcal{S}_{P, T}$ is the set of $\mathbf{w} \in \mathbb{R}^{s}$ such that there exists an $\mathbf{x} \in\left[\frac{\delta P^{k}}{2}, P^{k}\right]^{s}$ with $\mathbf{w}=V(\mathbf{x})$ and such that one has $\left|w_{i}\right|<\frac{\min \left(1, \Delta_{1}^{1 / R}\right)}{3 T}$ for $1 \leq i \leq r$ and $\left|w_{i}\right|<\frac{\min \left(1, \Delta_{1}^{1 / R}\right)}{3}$ for $r+1 \leq i \leq R$.

Now we give a lemma, which is essentially due to Nadesalingam and Pitman. (See [16], Lemma 5.2.) 
Lemma 8.8. Let $R$ and $s$ be positive integers satisfying $s>R$. Let

$$
A=\left(\lambda_{i j}\right)_{\substack{1 \leq i \leq R \\ 1 \leq j \leq s}}
$$

be a real $R \times s$ matrix. For $1 \leq i \leq R$, we define the linear forms $L_{i}(\mathbf{y})=\sum_{j=1}^{s} \lambda_{i j} y_{j}$. Let $\Delta_{1}$ denote the absolute value of the determinant of the left-hand $R \times R$ submatrix of $A$. Suppose that we have $\Delta_{1}>0$. Additionally, suppose that $Q$ is a real number satisfying

$$
Q \geq 6 s^{R} R^{2 R}\|A\|^{2 R} \Delta_{1}^{-2} .
$$

Suppose also that $w_{1}, \ldots, w_{R}$ are real numbers satisfying

$$
\left|w_{i}\right| \leq \frac{\Delta_{1}^{1 / R}}{3} \quad \text { for } \quad 1 \leq i \leq R
$$

Let $S_{0}=S_{0}\left(w_{1}, \ldots, w_{R}\right)$ be the set of all real vectors $\left(y_{R+1}, \ldots, y_{s}\right) \in[-Q, Q]^{s-R}$ for which there exist real numbers $y_{1}, \ldots, y_{R} \in[-Q, Q]$ with $w_{i}=L_{i}(\mathbf{y})$ for $1 \leq$ $i \leq R$.

Then $S_{0}$ has $(s-R)$-dimensional measure satisfying

$$
\mu_{s-R}\left(S_{0}\right) \gg_{A} Q^{s-R},
$$

where the implicit constant in Vinogradov's notation depends on $s$ and $R$ and the entries of $A$.

Proof. We apply the lemma of Nadesalingam and Pitman to the $R$ linear forms $M_{1}(\mathbf{y}), \ldots, M_{R}(\mathbf{y})$ defined by $M_{i}(\mathbf{y})=\Delta_{1}^{-1 / R} L_{i}(\mathbf{y})$ for $1 \leq i \leq R$, in order to relax the requirement $\Delta_{1} \geq 1$ of their lemma. We note that there is a slight difference between the definition of $\|A\|$ that we use and the definition they use, which accounts for the change in the condition (8.29). Here we have also implicitly used the last equation on page 704 of [15] to show that the term $H(L)$ in the lemma of Nadesalingam and Pitman is positive.

Now we return to the proof of Lemma 8.7 and apply Lemma 8.8. By 8.26) and the assumption $T \geq 1$, we may apply the lemma, with the choice $Q=\left(\delta P^{k}\right) / 2$, for any $\mathbf{w}=\left(w_{1}, w_{2}, \ldots, w_{R}\right)$ with

$\left|w_{i}\right|<\frac{\min \left(1, \Delta_{1}^{1 / R}\right)}{3 T}$ for $1 \leq i \leq r$, and $\left|w_{i}\right|<\frac{\min \left(1, \Delta_{1}^{1 / R}\right)}{3}$ for $r+1 \leq i \leq R$.

We obtain

$$
\mu_{s-R}\left(S_{0}\left(w_{1}, \ldots, w_{R}\right)\right) \gg_{\mathbf{F}, \delta} P^{k(s-R)} .
$$

Now, for any choice of $\left(y_{R+1}, y_{R+2}, \ldots, y_{s}\right) \in S_{0}\left(w_{1}, \ldots, w_{R}\right)$, there exist real numbers $y_{1}, \ldots, y_{R} \in[-Q, Q]$ with $L_{i}(\mathbf{y})=w_{i}$ for $1 \leq i \leq R$. Defining $\mathbf{z}$ as in (3.6), we have

$$
L_{i}\left(P^{k} \mathbf{z}+\mathbf{y}\right)=w_{i} \quad \text { for } \quad 1 \leq i \leq R .
$$

By (3.6) and our choice of $Q$, we also have

$$
P^{k} \mathbf{z}+\mathbf{y} \in\left[\frac{\delta}{2} P^{k}, \frac{1+\delta}{2} P^{k}\right] \subseteq\left[\frac{\delta}{2} P^{k}, P^{k}\right] .
$$


Recalling the definition of $\mathcal{S}_{P, T}$, we see that we have

$$
\mu_{s}\left(\mathcal{S}_{P, T}\right) \gg_{\mathbf{F}, \delta} T^{-r} P^{k(s-R)} .
$$

Combining with $(8.27)$ and $(\underline{8.28})$, we see that there is a positive real constant $c_{1}=c_{1}(\mathbf{F}, k, R, r, s, \delta, \mathbf{z})$ such that one has

$$
\mathcal{I}_{T}(P) \geq c_{1} P^{s-R k}
$$

This completes the proof of Lemma 8.7

Combining Lemmas 8.6 and 8.7 yields the following lower bound for $\mathcal{I}(P)$.

Lemma 8.9. Suppose that we are in the setting of Theorem 1.2 and that the coefficient matrix $A$ of the system $\mathbf{F}$ satisfies (3.2) and (3.3), and that there is a real vector $\mathbf{z}$ satisfying (3.6). Assume also that we have $\|\mathbf{F}\| \geq 1$. Define $\mathcal{I}(P)$ as in (8.6). Then there is a constant $c_{3}=c_{3}(\mathbf{F}, k, R, r, s, \delta, \mathbf{z})$ such that for $P \geq c_{3}$, one has

$$
\mathcal{I}(P) \gg P^{s-R k}
$$

Here the implicit constant in Vinogradov's notation may depend on $\mathbf{F}, k, s, R, r, \delta$ and the special real vector $\mathbf{z}$, but it does not depend on $P$.

8.4. Completion of the treatment of the major arcs. We wrap up our work on the major arcs with the following lemma.

Lemma 8.10. Suppose that we are in the setting of Theorem 1.2 and that the coefficient matrix $A$ of the system $\mathbf{F}$ satisfies (3.2) and (3.3), and that there is a real vector $\mathbf{z}$ satisfying (3.6). Assume also that we have $\|\mathbf{F}\| \geq 1$. Then there are constants $c_{4}$ and $c_{5}$, which may depend on $\mathbf{F}, k, s, R, r, \delta$ and $\mathbf{z}$, but which do not depend on $P$, such that for real numbers $P$ satisfying $P \geq c_{4}$, one has

$$
\int_{\mathcal{M}} \prod_{j=1}^{s} g_{j}(\boldsymbol{\alpha}) \prod_{i=r+1}^{R} K\left(\alpha_{i}\right) d \boldsymbol{\alpha} \geq c_{5} P^{s-R k}
$$

Proof. Choose $\epsilon=1 /(2 k)$ and apply Lemma 8.2. Since we have $\ell \geq 2 k+1$ and by the definition (4.10) of $B$, we obtain

$$
\int_{\mathcal{M}} \prod_{j=1}^{s} g_{j}(\boldsymbol{\alpha}) \prod_{i=r+1}^{R} K\left(\alpha_{i}\right) d \boldsymbol{\alpha}-\mathfrak{S I}(P) \ll P^{s-R k}(\log P)^{-1 /(8 k(R+1))} .
$$

Since condition (iv) of Theorem 1.2 holds, one has $\mathfrak{S} \gg 1$. By Lemma 8.9 there are constants $c_{3}$ and $c_{6}$ that do not depend on $P$ such that one has

$$
\mathcal{I}(P) \geq c_{6} P^{s-R k} \quad \text { for } \quad P \geq c_{3} \text {. }
$$

Lemma 8.10 follows from $(8.32$ and $(8.33)$ and the bound $\mathfrak{S} \gg 1$.

\section{Completion of the Proof of Theorem 1.2}

In this section, we gather together all of our results in order to complete the proof of Theorem 1.2

We recall that we demonstrated in Section 3 that we may assume that we have $\epsilon=1$, that we have $\|\mathbf{F}\| \geq 1$, that the coefficient matrix $A$ of the system $\mathbf{F}$ satisfies (3.2) and (3.3), and that there is a real vector $\mathbf{z}$ satisfying (3.6). 
We first observe how one proves the last sentence of Theorem 1.2, namely that if we have $r \geq 1$, and we define $m_{0}\left(r, k, \tilde{C}_{1}\right)$ as in (1.4) and assume that we have $\ell \geq m_{0}\left(r, k, \tilde{C}_{1}\right)$, then we have $\mathfrak{S} \gg_{\mathbf{F}} 1$. For $k \geq 3$, one simply applies Lemma 8.4. noting that we certainly have $\ell \geq 2 k+1$ for a sufficiently large choice of the constant $\tilde{C}_{1}$. For $k=2$, we may apply Lemma 8.5. Thus we have $\mathfrak{S} \gg_{\mathbf{F}} 1$.

Now we turn to the central result of Theorem 1.2. Recall from (4.9) that we have

$$
\mathcal{N}(P) \geq \int_{W} \prod_{j=1}^{s} g_{j}(\boldsymbol{\alpha}) \prod_{i=r+1}^{R} K\left(\alpha_{i}\right) d \boldsymbol{\alpha},
$$

where $\mathcal{N}(P)$ was defined to be the number of solutions of the system (4.1) with $x_{j} \in \mathcal{A}\left(P, P^{\eta}\right)$ for $1 \leq j \leq s$.

We first choose a function $T(P)$ as in Lemma 5.5. We can now treat the minor arcs and trivial arcs. By Lemmas [6.3 and 7.1, one obtains

$$
\int_{\mathfrak{m} \cup \mathfrak{t}} \prod_{j=1}^{s}\left|g_{j}(\boldsymbol{\alpha})\right| \prod_{i=r+1}^{R}\left|K\left(\alpha_{i}\right)\right| d \boldsymbol{\alpha}=o\left(P^{s-R k}\right) .
$$

We now consider the major arcs. By Lemma 8.10, we have

$$
\int_{\mathcal{M}} \prod_{j=1}^{s} g_{j}(\boldsymbol{\alpha}) \prod_{i=r+1}^{R} K\left(\alpha_{i}\right) d \boldsymbol{\alpha} \geq c_{5} P^{s-R k}
$$

for $P \geq c_{4}$, where $c_{4}$ and $c_{5}$ are constants that do not depend on $P$. Together with (9.2), it follows for sufficiently large $P$ that one has

$$
\int_{W} \prod_{j=1}^{s} g_{j}(\boldsymbol{\alpha}) \prod_{i=r+1}^{R} K\left(\alpha_{i}\right) d \boldsymbol{\alpha} \geq \frac{c_{5}}{2} P^{s-R k} .
$$

By (9.1), for sufficiently large $P$, we have

$$
\mathcal{N}(P) \gg_{\mathbf{F}, R, s, k} P^{s-R k} .
$$

This establishes Theorem 1.2

As a final observation, we note that we have obtained a lower bound of the expected order of magnitude for the number of solutions of our system in a box of size $P$, for all sufficiently large positive $P$. Recall that we assumed that we have $\epsilon=1$, that we have $\|\mathbf{F}\| \geq 1$, that the coefficient matrix $A$ of the system $\mathbf{F}$ satisfies (3.2) and (3.3), and that there is a real vector $\mathbf{z}$ satisfying (3.6). Using standard techniques, one can check that under the conditions of either Theorem 1.1 or Theorem 1.2 without any of these simplifying assumptions, the same lower bound holds for sufficiently large $P$. We note that in this case, $P$ must be sufficiently large also in terms of $\epsilon$, and the implicit constant in the lower bound for $\mathcal{N}(P)$ depends on $\epsilon$.

\section{REFERENCES}

1. M. Aigner, Combinatorial theory, Grundlehren der Mathematischen Wissenschaften, SpringerVerlag, New York/Heidelberg/Berlin, 1979. MR 80h:05002

2. R. C. Baker, Diophantine inequalities, London Mathematical Society Monographs, New Series, 1, The Clarendon Press, Oxford University Press, New York, 1986. MR 88f:11021 
3. V. Bentkus and F. Götze, Lattice point problems and distribution of values of quadratic forms, Ann. of Math. (2) 150 (1999), no. 3, 977-1027. MR 2001b:11087

4. B. J. Birch and H. Davenport, Indefinite quadratic forms in many variables, Mathematika, $\mathbf{5}$ (1958), 8-12. MR 20:3104

5. J. Brüdern and R. J. Cook, On simultaneous diagonal equations and inequalities, Acta Arith. 62 (1992), 125-149. MR 93h:11036

6. H. Davenport, Analytic methods for Diophantine equations and Diophantine inequalities, Ann Arbor Publishers, Ann Arbor, MI, 1963. MR 28:3002

7. H. Davenport and D. J. Lewis, Simultaneous equations of additive type, Philos. Trans. Roy. Soc. London Ser. A 264 (1969), 557-595. MR 39:6848

8. J. Edmonds, Minimum partition of a matroid into independent subsets, J. Res. Nat. Bureau Standards 69B (1965), 67-72. MR 32:7441

9. D. E. Freeman, A note on one cubic Diophantine inequality, J. London Math. Soc. (2), 61 (2000), no.1, 25-35. MR 2001c:11043

10. Luadratic Diophantine inequalities, J. Number Theory, 89 (2001), no.2, 268-307.

11. _ Asymptotic lower bounds for Diophantine inequalities, to appear in Mathematika.

12. $\ldots$ Asymptotic lower bounds and formulas for Diophantine inequalities, to appear in the Proceedings of the Millennial Conference in Number Theory.

13. L. Low, J. Pitman and A. Wolff, Simultaneous diagonal congruences, J. Number Theory 29 (1988), 31-59. MR 89g:11030

14. I. D. Meir, Simultaneous diagonal p-adic equations, Mathematika 45 (1998), 337-349. MR 2000k:11052

15. T. Nadesalingam and J. Pitman, Bounds for solutions of simultaneous diagonal equations of odd degree, Théorie des nombres (Québec, PQ, 1987), 703-734, de Gruyter, Berlin, 1989. MR 91f:11021

16. 118-158. MR 91c:11019

17. W. M. Schmidt, Diophantine inequalities for forms of odd degree, Adv. Math. 38 (1980), 128-151. MR 82h:10033

18. Simultaneous rational zeros of quadratic forms, Seminar Delange-Pisot-Poitou 1981. Progress in Math., Vol. 22, Birkhäuser, Boston, MA, 1982, 281-307. MR 84g:10041

19. R. C. Vaughan, On Waring's problem for cubes, J. Reine Angew. Math. 365 (1986), 122-170. MR 87j:11103

20. - On Waring's problem for smaller exponents. II, Mathematika 33 (1986), 6-22. MR 87j:11104

21. bridge University Press, Cambridge, U.K., 1997. MR 98a:11133

22. Y. Wang, Diophantine equations and inequalities in algebraic number fields, Springer-Verlag, Berlin/Heidelberg/New York, 1991. MR 92a:11036

23. T. D. Wooley, New estimates for smooth Weyl sums, J. London Math. Soc. (2) 51 (1995), 1-13. MR 96e:11109

Department of Mathematics, University of Colorado, 395 UCB, Boulder, Colorado 80309

Current address: School of Mathematics, Institute for Advanced Study, 1 Einstein Drive, Princeton, NJ 08540

E-mail address: freem@ias.edu 